\title{
Analytic Solutions of the Forces and Displacements for Multicentre Circular Arc Tunnels
}

\author{
Xingbo Han $\mathbb{D}^{1,2}$ and Yongxu Xia ${ }^{1}$ \\ ${ }^{1}$ School of Highway, Chang'an University, Xi'an, Shaanxi 710064, China \\ ${ }^{2}$ School of Civil and Environmental Engineering, The University of New South Wales, Sydney, NSW 2552, Australia
}

Correspondence should be addressed to Xingbo Han; xingbo.han@chd.edu.cn

Received 1 February 2018; Revised 26 March 2018; Accepted 8 April 2018; Published 16 May 2018

Academic Editor: Federico Guarracino

Copyright ( 2018 Xingbo Han and Yongxu Xia. This is an open access article distributed under the Creative Commons Attribution License, which permits unrestricted use, distribution, and reproduction in any medium, provided the original work is properly cited.

\begin{abstract}
A complex variable method for solving the forces and displacements for multicentre circular arc (MCA) tunnels by using analytical solutions is presented. The governing equations for the stresses and displacements are expressed in terms of series expression. Combined with the boundary conditions at lining inner region, lining-surrounding rock-mass interface, and infinity area, the undetermined coefficients of the analytic functions are obtained by solving a linear equation set. Forces solutions for linings are also presented according to the tangential stress at the two boundaries. Conformal mapping function for the MCA tunnel is given from the optimization method. Calculation results show that solutions with high accuracy can be obtained when the number of terms of power series is greater than 30. The effects of the degrees from the tunnel crown to the invert, coefficient of the lateral earth pressure, and distance from the rock-mass to the interface on regulations of the lining forces and rock-mass stresses are also thoroughly investigated.
\end{abstract}

\section{Introduction}

The design of the cross-section shape for transportation tunnels has undergone the development from a singer-centre circular arc to a multicentre circular arc (MCA). Though the singer-centred arc tunnel is simple for engineers to design, the MCA tunnel is widely used for its advantage in the reduction of the excavation volume. However, lining forces and displacements, which are essential parameters during every stages (i.e., design, construction, operation, and maintaining [1-3]) of the tunnel life circle, are difficult to analyse for a MCA tunnel. Therefore, suitable methods should be established to give access to the forces and displacements analysis of the MCA tunnels.

Very extensive numerical simulations have been performed to determine stresses and deformations of tunnels in recent year. Möller and Vermeer (2008) applied FEM to simulate lining forces and the ground deformation of the Steinhaldenfeld tunnel and Heinenoord tunnel [4]. Liu et al. (2017) simulated the whole construction stages of the Gongti North Road tunnel of Beijing Metro Line 10 built using the PBA method [5]. Also, Avgerinos et al. (2017) performed a series of three-dimensional finite-element analyses to discuss the forces, bending moment, and lining deformation of the future Crossrail tunnel [6]. However, these numerical results are mostly applied to certain examples and are difficult to give an understanding of how final solutions are influenced by different parameters. Behnen et al. (2015) pointed out that a design strategy based on comprehensible analytical models in combination with the valuable knowledge of experienced engineers should be preferred instead of relying on the complex simulation models [7].

Many researchers have treated the tunnel lining structures as shallow arches to present theoretical analysis models for its forces and displacements. Pi et al. (2001) applied the energy method to investigate the stresses and buckling of the shallow arches subjected to a radial load uniformly distributed around the arch axis [8]. Gao et al. (2014) combined the structure analysis and material uncertainties of arches and presented the results of the axial compressive force, bending moment, and axial displacement [9]. But these shallow arches are still not well satisfied with the tunnel lining boundary conditions in field. 


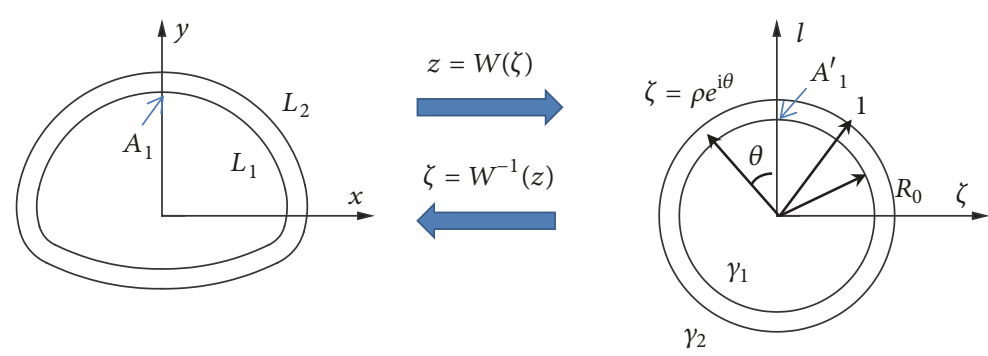

FigURE 1: Conformal mapping of tunnel cross-section in $z$-plane into $\zeta$-plane.

The theoretical methods for circular tunnels have also fully developed. Lekhnitskii (1968) proposed a complex variable-function method for the accurate solution of stresses around a circular hole under in-plane loading [10]. Hefny and Lo (1999) obtained analytical solutions for stresses and displacements around unlined circular tunnels excavated in elastic transversely isotropic [11]. Vu et al. (2013) developed a semianalytical solution for an unlined circular tunnel excavated in a transversely isotropic formation with nonlinear behaviour [12]. In addition, for lined circular tunnels [1315], ITA report (2000) recommended the elastic equation method to determine the member forces and cross-section deformation of the segmental ring [16]. Lu et al. (2011) applied the complex method and presented the stress solutions for a circular lined tunnel [17]. Yasuda et al. (2017) established a two-dimensional elastic solution for a deep circular tunnel under the far-field static loading [18]. It is still difficult to determine the accurate solutions for the stresses and displacements for the other irregular shapes.

An attempt is made in this study to find the forces and displacements solutions for MCA tunnel linings by applying Muskhelishvili's complex variable method.

\section{Complex Variable Method for Lined Noncircular Tunnels}

2.1. General Considerations. The problem can be considered as a reinforced hole in an infinite plane subjected to a uniform stress state at infinity, while the tunnel is located at a great depth compared with the tunnel dimension. The infinite plane on the complex plane is divided into two isotropic homogenous regions considering both the lining and the surrounding rock-mass. As shown in Figure 1, the two regions are bounded by contours $\gamma_{1}$ and $\gamma_{2}$, which represents the inner and outer boundaries of the lining, respectively. It is assumed that the conformal mapping function can be expressed as follows:

$$
z=\omega(\zeta)=R\left(\zeta+\sum_{k=0}^{n} c_{k} \zeta^{-k}\right)
$$

where $R$ is a real constant presenting the scale of the mapping.

The stress components provided by Muskhelishvili and Radok (1953) [19] based on the complex variable method is as follows:

$$
\sigma_{x}+\sigma_{y}=4 \operatorname{Re} \varphi^{\prime}(z)
$$

$$
\sigma_{y}-\sigma_{x}+2 i \tau_{x y}=2\left[\bar{z} \varphi^{\prime \prime}(z)+\psi^{\prime}(z)\right]
$$

where $\sigma_{x}$ and $\sigma_{y}$ are the horizontal and vertical stress components, respectively, $\tau_{x y}$ is the shear stress. $\varphi(z)$ and $\psi(z)$ are two analytic complex functions, and $\operatorname{Re}\{\}$ denotes the real part of \{\} .

The displacement can be described as follows:

$$
2 G(u+\mathrm{i} v)=\kappa \varphi(z)-z \overline{\varphi^{\prime}(z)}-\overline{\psi(z)}
$$

where $\mathrm{i}=\sqrt{-1}, G$ is the shear modulus, and $\kappa$ is a parameter related to Poisson's ratio $v$ and is as follows:

$$
\kappa= \begin{cases}3-4 v & \text { planestrian } \\ \frac{3-4 v}{1+v} & \text { planestress. }\end{cases}
$$

The surface tractions along the boundaries satisfy the following equation:

$$
\mathrm{i} \int\left(\bar{f}_{x}+\mathrm{i} \bar{f}_{y}\right) \mathrm{d} s=\varphi(z)+z \overline{\varphi^{\prime}(z)}+\overline{\psi(z)},
$$

where $\bar{f}_{x}$ and $\bar{f}_{y}$ are components of the surface traction vector in $x$ and $y$ directions, respectively.

The tunnel from the $z$-plane can be mapped into the $\zeta$-plane using (1). The analytic functions in $\zeta$-plane can be calculated by the following equations:

$$
\begin{aligned}
\varphi(\zeta) & =\varphi_{1}(z)=\varphi_{1}[\omega(\zeta)] \\
\psi(\zeta) & =\psi_{1}(z)=\psi_{1}[\omega(\zeta)] \\
\varphi_{1}^{\prime}(z) & =\frac{\varphi^{\prime}(\zeta)}{\omega^{\prime}(\zeta)} \\
\psi_{1}^{\prime}(z) & =\frac{\psi^{\prime}(\zeta)}{\omega^{\prime}(\zeta)} .
\end{aligned}
$$

Substituting (6) and (1) into (2), the stress components in the orthogonal curvilinear coordinate system can be rewritten as follows:

$$
\sigma_{\rho}+\sigma_{\theta}=\sigma_{x}+\sigma_{y}=4 \operatorname{Re}\left[\frac{\varphi^{\prime}(\zeta)}{\omega^{\prime}(\zeta)}\right]
$$




$$
\begin{aligned}
& \sigma_{\theta}-\sigma_{\rho}+2 \mathrm{i} \tau_{\rho \theta}=\frac{2 \zeta^{2}}{\rho^{2}} \\
& \cdot \frac{1}{\overline{\omega^{\prime}(\zeta)}}\left\{\overline{\omega(\zeta)} \frac{\varphi^{\prime \prime}(\zeta) \omega^{\prime}(\zeta)-\varphi^{\prime}(\zeta) \omega^{\prime \prime}(\zeta)}{\left[\omega^{\prime}(\zeta)\right]^{2}}\right. \\
& \left.+\psi^{\prime}(\zeta)\right\} .
\end{aligned}
$$

When the tunnel is unlined, the displacement of the surrounding rock-mass can be expressed as follows:

$$
2 G_{1}\left(u_{1}^{R}+\mathrm{i} v_{1}^{R}\right)=\kappa_{1} \varphi_{1}(\zeta)-\frac{\omega(\zeta)}{\overline{\omega^{\prime}(\zeta)}} \overline{\varphi_{1}^{\prime}(\zeta)}-\overline{\psi_{1}(\zeta)},
$$

where $u_{1}{ }^{R}$ and $v_{1}{ }^{R}$ are the displacement components in the $x$ and $y$ directions and $G_{1}$ and $\kappa_{1}$ refer to the parameters of the surrounding rock-mass.

Considering that the lining is installed when the displacement is $\eta$ times the total displacement, the displacement of the rock $u_{2}{ }^{R}+\mathrm{i} v_{2}{ }^{R}$ occurs before the support of the lining and can be expressed as follows:

$$
u_{2}^{R}+\mathrm{i} v_{2}^{R}=\eta\left(u_{1}^{R}+\mathrm{i} v_{1}^{R}\right) .
$$

After the lining is applied, a part of the displacement of the surrounding rock-mass is restricted and can be then presented as follows:

$$
2 G_{1}\left(u_{3}{ }^{R}+\mathrm{i} v_{3}{ }^{R}\right)=\kappa_{1} \varphi_{2}(\zeta)-\frac{\omega(\zeta)}{\overline{\omega^{\prime}(\zeta)}} \overline{\varphi_{2}^{\prime}(\zeta)}-\overline{\psi_{2}(\zeta)} .
$$

From (8) to (10), the final displacement of the surrounding rock-mass $u^{R}+\mathrm{i} v^{R}$ can be obtained as follows:

$$
\begin{aligned}
u^{R}+ & \mathrm{i} v^{R} \\
= & \left(u_{1}{ }^{R}+\mathrm{i} v_{1}{ }^{R}\right)-\left(u_{2}{ }^{R}+\mathrm{i} v_{2}{ }^{R}\right)+\left(u_{3}{ }^{R}+\mathrm{i} v_{3}^{R}\right) \\
= & \frac{1-\eta}{2 G_{1}}\left[\kappa_{1} \varphi_{1}(\sigma)-\frac{\omega(\sigma)}{\overline{\omega^{\prime}(\sigma)}} \overline{\varphi_{1}^{\prime}(\sigma)}-\overline{\psi_{1}(\sigma)}\right] \\
& +\frac{1}{2 G_{1}}\left[\kappa_{1} \varphi_{2}(\sigma)-\frac{\omega(\sigma)}{\overline{\omega^{\prime}(\sigma)}} \overline{\varphi_{2}^{\prime}(\sigma)}-\overline{\psi_{2}(\sigma)}\right] .
\end{aligned}
$$

The displacement of the lining can be derived by the following equation:

$$
2 G_{2}\left(u^{L}+i v^{L}\right)=\kappa_{2} \varphi_{3}(\zeta)-\frac{\omega(\zeta)}{\overline{\omega^{\prime}(\zeta)}} \overline{\varphi_{3}^{\prime}(\zeta)}-\overline{\psi_{3}(\zeta)},
$$

where $u^{L}$ and $v^{L}$ are the displacement components of any point of the lining in the $x$ and $y$ directions and $G_{2}$ and $\kappa_{2}$ refer to the parameters of the lining concrete.

The stress components of any point in the lining can be then calculated by substituting (13) into (7).

$$
\begin{aligned}
& \varphi(\zeta)=\varphi_{3}(\zeta) \\
& \psi(\zeta)=\psi_{3}(\zeta)
\end{aligned}
$$

In particular, the line $\rho=1$ denotes the interface of the surrounding rock mass and the lining. The line $\rho=R_{0}$ denotes the lining inner boundary.

Similarly, the stress components of any point in the surrounding rock mass can be solved by substituting (14) into (7).

$$
\begin{aligned}
& \varphi(\zeta)=\Gamma \omega(\zeta)+\varphi_{1}(\zeta)+\varphi_{2}(\zeta) \\
& \psi(\zeta)=\Gamma^{\prime} \omega(\zeta)+\psi_{1}(\zeta)+\psi_{2}(\zeta) .
\end{aligned}
$$

In (14), $\Gamma=p(1+\lambda) / 4$ and $\Gamma^{\prime}=p(\lambda-1) / 2$. The first term is the corresponding complex potential function before excavation, and the remaining terms represent the effects of the excavation and the lining support on the surrounding rock-mass.

The stresses of the lining and surrounding rock mass can be determined using (7) once the six analytic functions are determined. And then the displacement can be determined using (11) and (12).

2.2. Solutions for the Analytic Functions. The six analytic functions can be expressed by power series as [20]

$$
\begin{aligned}
\varphi_{1}(\zeta)= & \sum_{k=1}^{n} a_{k} \zeta^{-k} \\
\psi_{1}(\zeta)= & -\frac{\overline{\omega(1 / \zeta)}}{\omega^{\prime}(\zeta)} \varphi_{1}^{\prime}(\zeta)+\sum_{k=1}^{n-2} S_{k} \zeta^{k}+S_{0} \\
& -\frac{p R}{2}(1+\lambda) \zeta^{-1}+\frac{p R}{2}(1-\lambda) \sum_{k=1}^{n} c_{k} \zeta^{-k} \\
\varphi_{2}(\zeta)= & b_{0}+\sum_{k=1}^{\infty} b_{k} \zeta^{-k} \\
\psi_{2}(\zeta)= & d_{0}+\sum_{k=1}^{\infty} d_{k} \zeta^{-k} \\
\varphi_{3}(\zeta)= & p_{0}+\sum_{k=1}^{\infty} e_{k} \zeta^{-k}+\sum_{k=1}^{\infty} f_{k} \zeta^{k} \\
\psi_{3}(\zeta)= & q_{0}+\sum_{k=1}^{\infty} g_{k} \zeta^{-k}+\sum_{k=1}^{\infty} h_{k} \zeta^{k} .
\end{aligned}
$$

Assume that

$$
\begin{aligned}
& b(\zeta)=\sum_{k=1}^{\infty} b_{k} \zeta^{-k} \\
& d(\zeta)=\sum_{k=1}^{\infty} d_{k} \zeta^{-k}, \\
& e(\zeta)=\sum_{k=1}^{\infty} e_{k} \zeta^{-k} \\
& f(\zeta)=\sum_{k=1}^{\infty} f_{k} \zeta^{k},
\end{aligned}
$$




$$
\begin{aligned}
& g(\zeta)=\sum_{k=1}^{\infty} g_{k} \zeta^{-k} \\
& h(\zeta)=\sum_{k=1}^{\infty} h_{k} \zeta^{k} .
\end{aligned}
$$

The coefficients $a_{k}$ of the $\varphi_{1}(\zeta)$ can be represented by the following equations:

$$
a_{i}=-2 \Gamma R c_{i}, \quad i=n, n-1
$$

Let $\mathbf{a}=\left\{\begin{array}{lll}a_{1} & \cdots & a_{n-2}\end{array}\right\}^{\mathbf{T}} ; a_{1}, \ldots, a_{n-2}$ can be determined as follows:

$$
\mathbf{A}_{n-2 \times n-2} \mathbf{a}_{1 \times n-2}=\mathbf{B}_{1 \times n-2}
$$

when $i=j, A_{i j}=j L_{i+j+1}-1$, and if $i+j+1>n, A_{i j}=-1$; when $i \neq j, A_{i j}=j L_{i+j+1}$, and if $i+j+1>n, A_{i j}=0$.

$$
\begin{aligned}
& \mathbf{B}_{i} \\
& =\left[\begin{array}{lllll}
2 \Gamma R c_{1}+\Gamma^{\prime} R & 2 \Gamma R c_{2} & 3 \Gamma R c_{3} & \cdots & (n-2) \Gamma R c_{n-2}
\end{array}\right]^{T} .
\end{aligned}
$$

The coefficients $L_{k}$ can be computed by the following equations:

$$
\begin{aligned}
L_{n} & =c_{n} \\
L_{n-1} & =c_{n-1} \\
L_{n-j+1} & =\sum_{k=1}^{j-2}(j-1-k) c_{j-1-k} L_{n-k+1}+c_{n-j-1}, \\
& j=3, \ldots, n .
\end{aligned}
$$

Thus, the coefficients $a_{k}$ of analytic function $\varphi_{1}(\zeta)$ can be determined by combining (22)-(25).

For the analytic function $\psi_{1}(\zeta)$, the $S_{k}$ in (16) can be calculated by the following equations:

$$
S_{k}= \begin{cases}-\sum_{j=1}^{n-1} j L_{j+1} a_{j}, & k=0 \\ -\sum_{j=1}^{n-k-1} j L_{j+k+1} a_{j}, & k=1, \ldots, n-2 .\end{cases}
$$

The three boundary conditions used to solve the undetermined coefficients $\left(b_{0}, b_{k}, d_{0}, d_{k}, p_{0}, q_{0}, e_{k}, f_{k}, g_{k}, h_{k}\right)$ from the analytic functions $\left(\varphi_{2}(\zeta), \psi_{2}(\zeta), \varphi_{3}(\zeta), \psi_{3}(\zeta)\right)$ are listing:

(i) The surface tractions along the inner boundary of lining at $L_{1}$ are zero.

(ii) The surface tractions continue at the interface $L_{2}$ from the both surrounding rock mass side and lining side.

(iii) The displacement continues at the interface $L_{2}$ from the both surrounding rock mass side and lining side.
The surface traction condition at inner boundary $L_{1}$ can be expressed as follows:

$$
\varphi_{3}\left(\sigma_{1}\right)+\frac{\omega\left(\sigma_{1}\right)}{\overline{\omega^{\prime}\left(\sigma_{1}\right)}} \overline{\varphi_{3}^{\prime}\left(\sigma_{1}\right)}+\overline{\psi_{3}\left(\sigma_{1}\right)}=0 .
$$

The surface condition at the interface $L_{2}$ can be presented as follows:

$$
\begin{aligned}
\varphi_{2} & (\sigma)+\frac{\omega(\sigma)}{\overline{\omega^{\prime}(\sigma)}} \overline{\varphi_{2}^{\prime}(\sigma)}+\overline{\psi_{2}(\sigma)} \\
& =\varphi_{3}(\sigma)+\frac{\omega(\sigma)}{\overline{\omega^{\prime}(\sigma)}} \overline{\varphi_{3}^{\prime}(\sigma)}+\overline{\psi_{3}(\sigma)}
\end{aligned}
$$

The displacement condition at the interface $L_{2}$ can be expressed as follows:

$$
u^{R}+i v^{R}=u^{L}+i v^{L} .
$$

Substituting (11) and (12) into (29), the following equation can be obtained:

$$
\begin{aligned}
\frac{1-\eta}{2 G_{1}} & {\left[\kappa_{1} \varphi_{1}(\sigma)-\frac{\omega(\sigma)}{\overline{\omega^{\prime}(\sigma)}} \overline{\varphi_{1}^{\prime}(\sigma)}-\overline{\psi_{1}(\sigma)}\right] } \\
& +\frac{1}{2 G_{1}}\left[\kappa_{1} \varphi_{2}(\sigma)-\frac{\omega(\sigma)}{\overline{\omega^{\prime}(\sigma)}} \overline{\varphi_{2}^{\prime}(\sigma)}-\overline{\psi_{2}(\sigma)}\right] \\
= & \frac{1}{2 G_{2}}\left[\kappa_{2} \varphi_{3}(\sigma)-\frac{\omega(\sigma)}{\overline{\omega^{\prime}(\sigma)}} \overline{\varphi_{3}^{\prime}(\sigma)}-\overline{\psi_{3}(\sigma)}\right] .
\end{aligned}
$$

Two functions can be derived using (27) and (28) based on the Cauchy integration [20]:

$$
\begin{aligned}
& {\overline{\varphi^{\prime}}}_{3}\left(\frac{1}{\zeta}\right)=-\frac{\overline{\omega^{\prime}}(1 / \zeta)}{\omega\left(R_{0}^{2} \zeta\right)}\left[p_{0}+q_{0}+p_{1}\left(R_{0}^{2} \zeta\right)\right. \\
& \left.\quad+p_{2}\left(R_{0}^{2} \zeta\right)+q_{1}\left(\frac{1}{\zeta}\right)+q_{2}\left(\frac{1}{\zeta}\right)\right] \\
& {\overline{\varphi^{\prime}}}_{3}\left(\frac{1}{\zeta}\right)=\frac{\overline{\omega^{\prime}}(1 / \zeta)}{\omega(\zeta)}\left[b_{0}+d_{0}-p_{0}-q_{0}+b(\zeta)\right. \\
& \left.\quad-p_{1}(\zeta)-p_{2}(\zeta)-q_{1}\left(\frac{1}{\zeta}\right)-q_{2}\left(\frac{1}{\zeta}\right)+d\left(\frac{1}{\zeta}\right)\right] \\
& +d^{\prime}\left(\frac{1}{\zeta}\right) .
\end{aligned}
$$

Substituting (28) into (30), the following equation can be obtained:

$$
\begin{gathered}
\frac{1-\eta}{2 G_{1}}\left[\kappa_{1} \varphi_{1}(\sigma)-\frac{\omega(\sigma)}{\overline{\omega^{\prime}(\sigma)}} \overline{\varphi_{1}^{\prime}(\sigma)}-\overline{\psi_{1}(\sigma)}\right] \\
+\frac{\kappa_{1} G_{2}+G_{1}}{G_{1} G_{2}} \varphi_{2}(\sigma)+\frac{G_{1}-G_{2}}{G_{1} G_{2}} \frac{\omega(\sigma)}{\overline{\omega^{\prime}(\sigma)}} \overline{\varphi_{2}^{\prime}(\sigma)} \\
+\frac{G_{1}-G_{2}}{G_{1} G_{2}} \overline{\psi_{2}(\sigma)}=\frac{1+\kappa_{2}}{G_{2}} \varphi_{3}(\sigma) .
\end{gathered}
$$


Using the Cauchy integration, (33) can be expressed as follows:

$$
\begin{aligned}
- & \frac{(1-\eta) \kappa_{1}}{G_{1}} \varphi_{1}(\zeta)+\frac{(1-\eta)}{G_{1}} \sum_{k=1}^{n-2} S_{k} \zeta^{-k} \\
& -\frac{\kappa_{1} G_{2}+G_{1}}{G_{1} G_{2}} b(\zeta)-\frac{G_{1}-G_{2}}{G_{1} G_{2}} \sum_{k=1}^{n-2} T_{k} \zeta^{-k} \\
= & -\frac{1+\kappa_{2}}{G_{2}} p_{1}(\zeta) .
\end{aligned}
$$

Substituting $\zeta$ by $1 / \zeta$, the Cauchy integration of the conjugate of (34) can be expressed as follows:

$$
\begin{gathered}
\frac{(1-\eta)}{G_{1}}\left[\frac{\bar{\omega}(\zeta)}{\omega^{\prime}(1 / \zeta)} \varphi_{1}^{\prime}\left(\frac{1}{\zeta}\right)-\sum_{k=1}^{n-2} S_{k} \zeta^{-k}-S_{0}\right. \\
\left.+\psi_{1}\left(\frac{1}{\zeta}\right)\right]-\frac{G_{1}-G_{2}}{G_{1} G_{2}}\left[\frac{\bar{\omega}(\zeta)}{\omega^{\prime}(1 / \zeta)} b^{\prime}\left(\frac{1}{\zeta}\right)\right. \\
\left.-\sum_{k=1}^{n-2} T_{k} \zeta^{-k}-T^{\prime}+d\left(\frac{1}{\zeta}\right)\right]=-\frac{1+\kappa_{2}}{G_{2}} p_{2}(\zeta)
\end{gathered}
$$

Substituting (16) into (35), the following equation can be derived:

$$
\begin{aligned}
& \frac{p R(1-\eta)}{2 G_{1}}\left[-(1+\lambda) \zeta+(1-\lambda) \sum_{k=1}^{n} c_{k} \zeta^{k}\right] \\
& -\frac{G_{1}-G_{2}}{G_{1} G_{2}}\left[\frac{\bar{\omega}(\zeta)}{\omega^{\prime}(1 / \zeta)} b^{\prime}\left(\frac{1}{\zeta}\right)-\sum_{k=1}^{n-2} T_{k} \zeta^{-k}-T^{\prime}\right. \\
& \left.+d\left(\frac{1}{\zeta}\right)\right]=-\frac{1+\kappa_{2}}{G_{2}} p_{2}(\zeta) .
\end{aligned}
$$

Then (36) can be expressed as follows [20]:

$$
\begin{aligned}
& \frac{p R(1-\eta)}{2 G_{1}}\left[-(1+\lambda) \zeta+(1-\lambda) \sum_{k=1}^{n} c_{k} \zeta^{k}\right] \\
& -\frac{G_{1}-G_{2}}{G_{1} G_{2}}\left[\sum_{k=2}^{\infty} T_{k}^{\prime} \zeta^{k-1}+d\left(\frac{1}{\zeta}\right)\right] \\
& =-\frac{1+\kappa_{2}}{G_{2}} p_{2}(\zeta) .
\end{aligned}
$$

The following equation can be obtained by combining (33)-(35):

$$
\begin{aligned}
& \frac{\kappa_{1} G_{2}+G_{1}}{G_{1} G_{2}} b_{0}+\frac{G_{1}-G_{2}}{G_{1} G_{2}} d_{0} \\
& =\frac{(1-\eta)}{G_{1}} S_{0}^{\prime}-\frac{G_{1}-G_{2}}{G_{1} G_{2}} T_{1}^{\prime}+\frac{1+\kappa_{2}}{G_{2}} p_{0} .
\end{aligned}
$$

The coefficients $T_{k}$ and $T_{k}^{\prime}$ in (34)-(38) can be calculated using the following equations:

$$
\begin{aligned}
& T_{k}=-\sum_{j=1}^{n-k-1} j L_{j+k+1} b_{j}, \quad k=1, \ldots, n-2 \\
& T_{1}^{\prime}=-\sum_{j=1}^{n-1} j L_{j+1} b_{j} \\
& T_{2}^{\prime}=-\sum_{j=1}^{n} j L_{j} b_{j} \\
& T_{k}^{\prime}=-\sum_{j=1}^{k-2} j L_{k-j-1} b_{j}-\sum_{j=k-1}^{n+k-2} j L_{j-k+2} b_{j}, \quad k=3, \ldots, \infty .
\end{aligned}
$$

The undetermined coefficients in $b(\zeta), d(\zeta), e(\zeta), f(\zeta)$, $g(\zeta)$, and $h(\zeta)$ can be determined based on (31), (32), (34), and (38). Linear simultaneous equations, which contain infinite number of equations, can be obtained by comparing the coefficient of the same order of variables $\zeta^{k}$. But only finite terms of $b(\zeta), d(\zeta), e(\zeta), f(\zeta), g(\zeta)$, and $h(\zeta)$ are discussed here in order to get the problem solved. The number of terms of $b_{k}, e_{k}$, and $h_{k}$ is denoted as $N e$, and number of terms of $d_{k}$, $f_{k}$, and $g_{k}$ are denoted as $N f$.

Let $p_{0}=x_{1}, e_{j}=x_{j+1}(j=1, \ldots, N e), q_{0}=x_{N e+2}, h_{j}=$ $x_{\mathrm{Ne}+2+j}(j=1, \ldots, \mathrm{Ne}), f_{k}=x_{2 \mathrm{Ne}+2+k}$, and $g_{k}=x_{2 \mathrm{Ne}+\mathrm{N} f+2+k}$ $(j=1, \ldots, N f)$; the following linear simultaneous equations can be obtained by comparing the constant and coefficients of the positive power of $\zeta^{k}$ in (31):

$$
\begin{aligned}
C_{i j} x_{j} & +C_{i(\mathrm{Ne}+1+k)} x_{\mathrm{Ne}+1+k}+C_{i(2 \mathrm{Ne}+2+L)} x_{2 \mathrm{Ne+2+L}} \\
& +C_{i(2 \mathrm{Ne}+\mathrm{N} f+2+L)} x_{2 \mathrm{Ne}+\mathrm{N} f+2+L}=0 .
\end{aligned}
$$

$\mathrm{Ne}+2$ equations are included in (41).

Comparing negative powers of $\zeta^{k}$ in (31), the following linear simultaneous equations can be obtained:

$$
\begin{gathered}
D_{i j} x_{j}+D_{i(\mathrm{Ne}+1+k)} x_{N e+1+k}+D_{i(2 \mathrm{Ne}+2+L)} x_{2 \mathrm{Ne+2+L}} \\
+D_{i(2 \mathrm{Ne}+\mathrm{N} f+2+L)} x_{2 \mathrm{Ne}+\mathrm{N} f+2+L}=0 .
\end{gathered}
$$

$N f-1$ equations are included in (42).

Let $b_{j}=x_{2 N e+2 N f+2+j}(j=1, \ldots, N e), d_{0}=x_{3 N e+2 N f+3}$, and $d_{j}=x_{3 N e+2 N f+3+j}(j=1, \ldots, N f)$; the following linear simultaneous equations can be obtained by comparing the constant and the coefficients of the positive power of $\zeta^{k}$ in (32):

$$
\begin{aligned}
E_{i j} x_{j} & +E_{i(N e+1+k)} x_{N e+1+k}+E_{i(2 N e+2+L)} x_{2 N e+2+L} \\
& +E_{i(2 N e+N f+2+L)} x_{2 N e+N f+2+L} \\
& +E_{i(2 N e+2 N f+2+k)} x_{2 N e+2 N f+2+k} \\
& +E_{i(3 N e+2 N f+2+M)} x_{3 N e+2 N f+2+M}=0 .
\end{aligned}
$$

$\mathrm{Ne}+2$ equations are included in (43). 
Comparing negative powers of $\zeta^{k}$ in (32), the following linear simultaneous equations can be obtained:

$$
\begin{aligned}
F_{i j} x_{j} & +F_{i(\mathrm{Ne}+1+k)} x_{\mathrm{Ne+1+k}}+F_{i(2 \mathrm{Ne}+2+L)} x_{2 \mathrm{Ne}+2+L} \\
& +F_{i(2 \mathrm{Ne}+\mathrm{N} f+2+L)} x_{2 \mathrm{Ne}+\mathrm{N} f+2+L} \\
& +F_{i(2 \mathrm{Ne}+2 \mathrm{~N} f+2+k)} x_{2 N e+2 N f+2+k} \\
& +F_{i(3 N e+2 N f+2+M)} x_{3 N e+2 N f+2+M}=0 .
\end{aligned}
$$

$N f-1$ equations are included in (44).

Comparing the coefficients of the negative powers of $\zeta^{k}$ in (34), the linear simultaneous equations can be derived as follows:

$$
G_{i j} x_{j}+G_{i(2 N e+2 N f+2+k)} x_{2 N e+2 N f+2+k}=H_{i} .
$$

$N f-1$ equations are included in (45).

$H_{i}$ in (45) can be expressed as

$$
H_{i}= \begin{cases}-(1-\eta) S_{i}+(1-\eta) \kappa_{1} a_{i} & i=1, \ldots, n-2 \\ (1-\eta) \kappa_{1} a_{i} & i=n-1, n .\end{cases}
$$

Comparing the coefficients of the negative powers of $\zeta^{k}$ in (37), the linear simultaneous equations can be obtained as

$$
\begin{gathered}
P_{i(2 \mathrm{Ne}+2+j)} x_{2 \mathrm{Ne}+2+j}+P_{i(2 \mathrm{Ne}+\mathrm{N} f+2+k)} x_{2 \mathrm{Ne}+\mathrm{N} f+2+k} \\
+P_{i(3 \mathrm{Ne}+2 \mathrm{~N} f+2+L)} x_{3 \mathrm{Ne}+2 \mathrm{~N} f+2+L}=Q_{i} .
\end{gathered}
$$

$N f-1$ equations are included in (47), where

$$
\begin{aligned}
& Q_{i} \\
& \quad= \begin{cases}\frac{p R(1-\eta)}{2}\left[(1+\lambda)-(1-\lambda) c_{1}\right] & i=1 \\
-\frac{p R(1-\eta)}{2}(1-\lambda) c_{i} & i=2, \ldots, n .\end{cases}
\end{aligned}
$$

Therefore, there are totally $3 N e+3 N f+2$ equations in (41) $-(48)$ for the $3 N e+3 N f+3$ undetermined variables. One more equation is still needed to solve all the variables.

The support effect of the lining to the rock-mass decreases as the distance between them increases. Thus, (10) can be expressed at infinity as

$$
\begin{aligned}
\lim _{\zeta \rightarrow \infty}\left[2 G_{1}\left(u_{3}{ }^{R}+\mathrm{i} v_{3}{ }^{R}\right)\right] \\
\quad=\lim _{\zeta \rightarrow \infty}\left[\kappa_{1} \varphi_{2}(\zeta)-\frac{\omega(\zeta)}{\overline{\omega^{\prime}(\zeta)}} \overline{\varphi_{2}^{\prime}(\zeta)}-\overline{\psi_{2}(\zeta)}\right]=0 .
\end{aligned}
$$

It can be obtained from (49) that

$\kappa_{1} \lim _{\zeta \rightarrow \infty} \varphi_{2}(\zeta)-\lim _{\zeta \rightarrow \infty}\left[\frac{\omega(\zeta)}{\overline{\omega^{\prime}(\zeta)}} \overline{\varphi_{2}^{\prime}(\zeta)}\right]-\lim _{\zeta \rightarrow \infty} \overline{\psi_{2}(\zeta)}=0$

And then

$$
\kappa_{1} b_{0}-d_{0}=0
$$

Substituting (51) and (40) into (38), the following equations can be obtained:

$$
\begin{aligned}
p_{0} & -\frac{1+\kappa_{1}}{1+\kappa_{2}} d_{0}+\frac{G_{1}-G_{2}}{G_{1}\left(1+\kappa_{2}\right)} \sum_{j=1}^{n-1} j L_{j+1} b_{j} \\
& =-\frac{G_{2}(1-\eta)}{G_{1}\left(1+\kappa_{2}\right)} S_{0}^{\prime}
\end{aligned}
$$

Now, there are totally $3 N e+3 N f+3$ equations for the $3 N e+3 N f+3$ variables. Briefly, the $x_{j}$ can be calculated using the following equation set:

$$
\left[\begin{array}{c}
{[C]_{(\mathrm{Ne}+2) \times(2 \mathrm{Ne}+2 \mathrm{Nf}+2)}} \\
{[D]_{(\mathrm{Nf}-1) \times(2 \mathrm{Ne}+2 \mathrm{Nf}+2)}} \\
{[E]_{(\mathrm{Ne}+2) \times(3 \mathrm{Ne}+3 N f+3)}} \\
{[F]_{(\mathrm{Nf}-1) \times(2 \mathrm{Ne}+2 \mathrm{Nf}+2)}} \\
{[G]_{(\mathrm{Ne}) \times(3 \mathrm{Ne}+2 \mathrm{Nf}+2)}} \\
{[P]_{(\mathrm{Nf}) \times(3 \mathrm{Ne}+3 \mathrm{Nf}+3)}} \\
1+\kappa_{2}
\end{array}\right]\left[[x]_{j \times 1}\right]
$$

$$
=\left[\begin{array}{c}
{[\mathbf{0}]_{(2 N e+2 N f+2) \times 1}} \\
{[H]_{N e \times 1}} \\
{[Q]_{N f \times 1}} \\
-\frac{G_{2}(1-\eta) S_{0}^{\prime}}{G_{1}}
\end{array}\right] \text {. }
$$

The coefficients of $C_{i j}, D_{i j}, E_{i j}, F_{i j}, G_{i j}$, and $P_{i j}$ have been listed in [16].

Thus, all the undetermined coefficients of analytic functions can be solved.

\section{Conformal Mapping for the MCA Tunnel}

The tunnel lining and rock-mass in $z$-plane can be mapped into an annulus in the $\zeta$-plane by (1). The interface of the lining and surrounding rock-mass is reflected by the external radius $\left(r_{1}=1\right)$. The inner boundary of the lining is reflected by the inside radius $\left(r_{0}<1\right)$. The undetermined parameters in (1) are $R$ and $c_{k}$.

Assume that any point $A_{j}$ at the inner boundary of lining in $z$-plane is transferred to the point $A^{\prime}{ }_{j}$ at the inside radius in $\zeta$-plane (Figure 1 ). Any point $B_{j}$ at the interface is similar to point $B^{\prime}{ }_{j}$ at the external radius. The conformal mapping function at the interface and the lining inner boundary can be expressed as follows:

$$
\begin{aligned}
& r_{A j}=R\left(r_{0} e^{\mathrm{i}\left(\beta_{A j}-\alpha_{A j}\right)}+\sum_{k=0}^{n} c_{k} r_{0}^{-k} e^{-\mathrm{i}\left(k \beta_{A j}+\alpha_{A j}\right)}\right) \\
& r_{B j}=R\left(r_{1} e^{\mathrm{i}\left(\beta_{B j}-\alpha_{B j}\right)}+\sum_{k=0}^{n} c_{k} r_{1}^{-k} e^{-\mathrm{i}\left(k \beta_{B j}+\alpha_{B j}\right)}\right) .
\end{aligned}
$$


Departing the real part and imaginary part, (54) can be then expressed as

$$
\begin{aligned}
& \sin \left(\beta_{A j}-\alpha_{A j}\right)-\sum_{k=0}^{n} c_{k} r_{0}{ }^{-k} \sin \left(k \beta_{A j}+\alpha_{A j}\right)=0 \\
& r_{A j} \\
& =R\left[\cos \left(\beta_{A j}-\alpha_{A j}\right)+\sum_{k=0}^{n} c_{k} r_{0}^{-k} \cos \left(k \beta_{A j}+\alpha_{A j}\right)\right] \\
& \sin \left(\beta_{B j}-\alpha_{B j}\right)-\sum_{k=0}^{n} c_{k} r_{1}^{-k} \sin \left(k \beta_{B j}+\alpha_{B j}\right)=0
\end{aligned}
$$

$$
\begin{aligned}
& r_{B j} \\
& =R\left[\cos \left(\beta_{B j}-\alpha_{B j}\right)+\sum_{k=0}^{n} c_{k} r_{1}^{-k} \cos \left(k \beta_{B j}+\alpha_{B j}\right)\right] .
\end{aligned}
$$

Suppose that $A_{1}$ can be accurately mapped into the point $A^{\prime}{ }_{1}, R$ can be determined as follows:

$$
R=\frac{r_{0}}{1+\sum_{k=0}^{n} c_{k} / r_{1}{ }^{k}} .
$$

$c_{k}$ and $r_{0}$ in (56) can be obtained by solving the following optimization problem:

Objective function:

$\min f$

$$
\begin{aligned}
= & \sum_{j=0}^{m}\left\{r^{*}{ }_{A j}-R\left[\cos \left(\beta_{A j}-\alpha_{A j}\right)+\sum_{k=0}^{n} \frac{c_{k} \cos \left(k \beta_{A j}+\alpha_{A j}\right)}{r_{0}}\right]\right\}^{2} \\
& +\sum_{j=0}^{m}\left\{r^{*}{ }_{B j}-R\left[\cos \left(\beta_{B j}-\alpha_{B j}\right)+\sum_{k=0}^{n} c_{k} \cos \left(k \beta_{B j}+\alpha_{B j}\right) r_{1}{ }^{-k}\right]\right\}^{2} .
\end{aligned}
$$

Boundary condition:

$$
\sum_{k=0}^{n}\left|k c_{k}\right|<1
$$

The cross-section of one common MCA highway tunnel is shown in Figure 2(a). Using the above method, the conformal mapping function can be expressed as follows:

$$
\begin{aligned}
z= & 5.7577\left(\zeta+0.22694-0.13876 \zeta^{-1}+0.0578 \zeta^{-2}\right. \\
& \left.-0.00958 \zeta^{-3}-0.00504 \zeta^{-4}\right) \\
R_{0} & =0.935 .
\end{aligned}
$$

As shown in Figure 2(b), the lining boundaries before and after mapping have a good agreement.

\section{Solutions for the Forces and Displacement}

4.1. Solutions for Stresses of Lining and Rock Mass. Combining (7) with (13), the stress components for any points of the lining can be obtained by the following equations:

$$
\begin{gathered}
\sigma_{\rho}+\sigma_{\theta}=4 \operatorname{Re}\left[\frac{\varphi_{3}^{\prime}(\zeta)}{\omega^{\prime}(\zeta)}\right] \\
\sigma_{\theta}-\sigma_{\rho}+2 \mathrm{i} \tau_{\rho \theta}=\frac{2 \zeta^{2}}{\rho^{2}}
\end{gathered}
$$

$$
\begin{aligned}
& \cdot \frac{1}{\overline{\omega^{\prime}(\zeta)}}\left\{\overline{\omega(\zeta)} \frac{\varphi_{3}^{\prime \prime}(\zeta) \omega^{\prime}(\zeta)-\varphi_{3}^{\prime}(\zeta) \omega^{\prime \prime}(\zeta)}{\left[\omega^{\prime}(\zeta)\right]^{2}}\right. \\
& \left.+\psi_{3}^{\prime}(\zeta)\right\} .
\end{aligned}
$$

Moreover, (60) can be expanded as follows:

$$
\begin{aligned}
\sigma_{\theta} & =2 \operatorname{Re}\left[\frac{\varphi_{3}^{\prime}(\zeta)}{\omega^{\prime}(\zeta)}\right]+\operatorname{Re}\left\{\frac{\zeta^{2}}{\rho^{2}}\right. \\
& \cdot \frac{1}{\overline{\omega^{\prime}(\zeta)}}\left[\overline{\omega(\zeta)} \frac{\varphi^{\prime \prime}{ }_{3}(\zeta) \omega^{\prime}(\zeta)-\varphi_{3}^{\prime}(\zeta) \omega^{\prime \prime}(\zeta)}{\omega^{\prime}(\zeta)^{2}}\right. \\
& \left.\left.+\psi_{3}^{\prime}(\zeta)\right]\right\} \\
\sigma_{\rho} & =2 \operatorname{Re}\left[\frac{\varphi_{3}^{\prime}(\zeta)}{\omega^{\prime}(\zeta)}\right]-\operatorname{Re}\left\{\frac{\zeta^{2}}{\rho^{2}}\right. \\
& \cdot \frac{1}{\overline{\omega^{\prime}(\zeta)}}\left[\overline{\omega(\zeta)} \frac{\varphi^{\prime \prime}{ }_{3}(\zeta) \omega^{\prime}(\zeta)-\varphi_{3}^{\prime}(\zeta) \omega^{\prime \prime}(\zeta)}{\omega^{\prime}(\zeta)^{2}}\right. \\
& \left.\left.+\psi_{3}^{\prime}(\zeta)\right]\right\}
\end{aligned}
$$



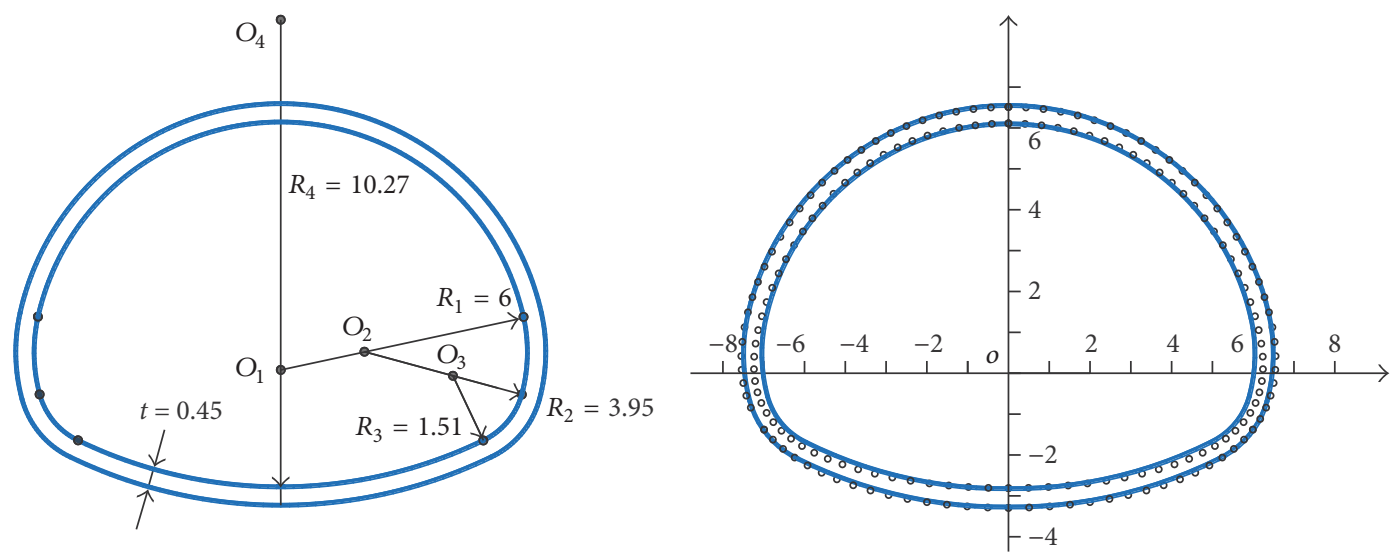

○० Mapped boundaries

— Designed boundaries

(a)

(b)

FIgURE 2: Tunnel cross-section before and after mapping (unit m).

$$
\begin{aligned}
\tau_{\rho \theta} & =\operatorname{Im}\left\{\frac{\zeta^{2}}{\rho^{2}}\right. \\
& \cdot \frac{1}{\overline{\omega^{\prime}(\zeta)}}\left[\frac{}{\omega(\zeta)} \frac{\varphi^{\prime \prime}{ }_{3}(\zeta) \omega^{\prime}(\zeta)-\varphi_{3}^{\prime}(\zeta) \omega^{\prime \prime}(\zeta)}{\omega^{\prime}(\zeta)^{2}}\right. \\
& \left.\left.+\psi_{3}^{\prime}(\zeta)\right]\right\} .
\end{aligned}
$$

In particular, the stresses of the interface can be calculated when $\rho=1$ and the stresses of the inner boundary of the lining can be calculated when $\rho=R_{0}$.

Combining (7) with (14), the stress components for points in rock-mass can be obtained by the following equations:

$$
\begin{aligned}
\sigma_{\theta} & =2 \operatorname{Re}\left[\frac{\varphi^{\prime}(\zeta)}{\omega^{\prime}(\zeta)}\right]+\operatorname{Re}\left\{\frac{\zeta^{2}}{\rho^{2}}\right. \\
& \cdot \frac{1}{\overline{\omega^{\prime}(\zeta)}}\left[\frac{\bar{\omega}(\zeta)}{\varphi^{\prime \prime}(\zeta) \omega^{\prime}(\zeta)-\varphi^{\prime}(\zeta) \omega^{\prime \prime}(\zeta)}\right. \\
& \left.\left.+\psi^{\prime}(\zeta)\right]\right\} \\
\sigma_{\rho}(\zeta)^{2} & =2 \operatorname{Re}\left[\frac{\varphi^{\prime}(\zeta)}{\omega^{\prime}(\zeta)}\right]-\operatorname{Re}\left\{\frac{\zeta^{2}}{\rho^{2}}\right. \\
& \cdot \frac{1}{\overline{\omega^{\prime}(\zeta)}}\left[\frac{\omega^{\prime \prime}(\zeta)}{\omega^{\prime \prime}(\zeta) \omega^{\prime}(\zeta)-\varphi^{\prime}(\zeta) \omega^{\prime \prime}(\zeta)}\right. \\
& \left.\left.+\psi^{\prime}(\zeta)\right]\right\} \\
\tau_{\rho \theta} & =\operatorname{Im}\left\{\frac{\zeta^{\prime}(\zeta)^{2}}{\rho^{2}}\right.
\end{aligned}
$$

$$
\begin{aligned}
& \cdot \frac{1}{\overline{\omega^{\prime}(\zeta)}}\left[\overline{\omega(\zeta)} \frac{\varphi^{\prime \prime}(\zeta) \omega^{\prime}(\zeta)-\varphi^{\prime}(\zeta) \omega^{\prime \prime}(\zeta)}{\omega^{\prime}(\zeta)^{2}}\right. \\
& \left.\left.+\psi^{\prime}(\zeta)\right]\right\} .
\end{aligned}
$$

The related terms in (61)-(62) can be calculated by the following equations:

$$
\begin{aligned}
\overline{\omega(\zeta)} & =\bar{\omega}(\bar{\zeta})=\bar{\omega}\left(\frac{\rho^{2}}{\zeta}\right) \\
& =R\left(\rho^{2} \zeta^{-1}+\sum_{k=0}^{n} c_{k} \rho^{-2 k} \zeta^{k}\right) \\
\bar{\omega}\left(\frac{1}{\zeta}\right) & =R\left(\zeta^{-1}+\sum_{k=0}^{n} c_{k} \zeta^{k}\right) \\
{\left[\bar{\omega}\left(\frac{1}{\zeta}\right)\right]^{\prime} } & =R\left(-\zeta^{-2}+\sum_{k=1}^{n} k c_{k} \zeta^{k-1}\right) \\
\omega^{\prime}(\zeta) & =R\left(1-\sum_{k=1}^{n} k c_{k} \zeta^{-k-1}\right) \\
\overline{\omega^{\prime}(\zeta)} & =R\left(1-\sum_{k=1}^{n} k c_{k} \rho^{-2 k-2} \zeta^{k+1}\right) \\
\omega^{\prime \prime}(\zeta) & =R \sum_{k=1}^{n} k(k+1) c_{k} \zeta^{-k-2}
\end{aligned}
$$

4.2. Solutions for Internal Forces. Assume that the tangential stress at different depth of the lining varies linearly along the lining thickness, the bending moment, and axial force of the lining can be obtained based on $\sigma_{\theta}$ of the inner and 


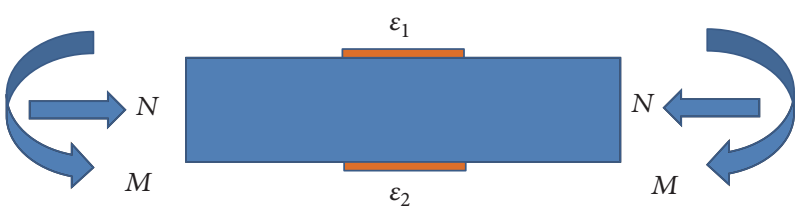

FIGURE 3: Sketch map for lining forces.

outer boundaries of the lining. The strains under the two boundaries of the lining caused by the bending moment and axial force are shown in Figure 3.

Denote that the strain is caused by the bending moment as $\varepsilon_{M}$ and axial force as $\varepsilon_{N}$. Strains at the two boundaries can be expressed as

$$
\begin{aligned}
& \varepsilon_{1}=\varepsilon_{N}-\varepsilon_{M} \\
& \varepsilon_{2}=\varepsilon_{N}+\varepsilon_{M} .
\end{aligned}
$$

Furthermore, $\varepsilon_{M}$ and $\varepsilon_{N}$ can be calculated by the following equations:

$$
\begin{gathered}
\varepsilon_{M}=\frac{\left(\varepsilon_{2}-\varepsilon_{1}\right)}{2} \\
\varepsilon_{N}=\frac{\left(\varepsilon_{2}+\varepsilon_{1}\right)}{2} .
\end{gathered}
$$

According to the material mechanics, the stress can be obtained as follows:

$$
\begin{gathered}
\sigma=\frac{M}{W} \\
\sigma=\frac{N}{A},
\end{gathered}
$$

where, $W$ is the lining bending rigidity and $A$ is the crosssection area.

Combining (64)-(66), the bending moment and axial force can be expressed as

$$
\begin{aligned}
& M=E_{2} \frac{\varepsilon_{2}-\varepsilon_{1}}{2} W=\frac{\sigma_{\theta 2}-\sigma_{\theta 1}}{2} W \\
& N=E_{2} \frac{\varepsilon_{2}+\varepsilon_{1}}{2} A=\frac{\sigma_{\theta 2}+\sigma_{\theta 1}}{2} A,
\end{aligned}
$$

where $\sigma_{\theta 1}$ is the Tangential stress on the tension side, while $\sigma_{\theta 2}$ is the Tangential stress on the compression side.

4.3. Solutions for Displacements. From (12), the displacement components of the lining can be expressed as follows:

$$
\begin{aligned}
& u^{L}=\operatorname{Re}\left\{\frac{1}{2 G_{2}}\left[\kappa_{2} \varphi_{3}(\zeta)-\frac{\omega(\zeta)}{\overline{\omega^{\prime}(\zeta)}} \overline{\varphi_{3}^{\prime}(\zeta)}-\overline{\psi_{3}^{\prime}(\zeta)}\right]\right\} \\
& v^{L}=\operatorname{Im}\left\{\frac{1}{2 G_{2}}\left[\kappa_{2} \varphi_{3}(\zeta)-\frac{\omega(\zeta)}{\overline{\omega^{\prime}(\zeta)}} \overline{\varphi_{3}^{\prime}(\zeta)}-\overline{\psi_{3}^{\prime}(\zeta)}\right]\right\} .
\end{aligned}
$$

From (11), the displacement components of the surrounding rock-mass can be expressed as

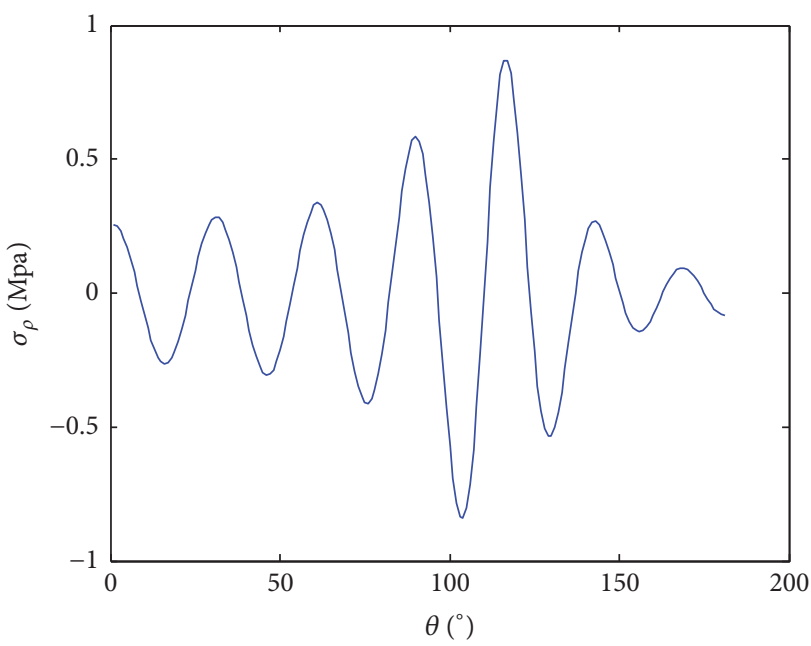

FIGURE 4: Normal stress of lining inner boundary $(\mathrm{Ne}=10)$.

$$
\begin{aligned}
u^{R} & =\operatorname{Re}\left\{\frac{1-\eta}{2 G_{1}}\left[\kappa_{1} \varphi_{1}(\sigma)-\frac{\omega(\sigma)}{\overline{\omega^{\prime}(\sigma)}} \overline{\varphi_{1}^{\prime}(\sigma)}-\overline{\psi_{1}(\sigma)}\right]\right. \\
& \left.+\frac{1}{2 G_{1}}\left[\kappa_{1} \varphi_{2}(\sigma)-\frac{\omega(\sigma)}{\overline{\omega^{\prime}(\sigma)}} \overline{\varphi_{2}^{\prime}(\sigma)}-\overline{\psi_{2}(\sigma)}\right]\right\} \\
v^{R} & =\operatorname{Im}\left\{\frac{1-\eta}{2 G_{1}}\left[\kappa_{1} \varphi_{1}(\sigma)-\frac{\omega(\sigma)}{\overline{\omega^{\prime}(\sigma)}} \overline{\varphi_{1}^{\prime}(\sigma)}-\overline{\psi_{1}(\sigma)}\right]\right. \\
& \left.+\frac{1}{2 G_{1}}\left[\kappa_{1} \varphi_{2}(\sigma)-\frac{\omega(\sigma)}{\overline{\omega^{\prime}(\sigma)}} \overline{\varphi_{2}^{\prime}(\sigma)}-\overline{\psi_{2}(\sigma)}\right]\right\} .
\end{aligned}
$$

\section{Example and Discussion}

Take a highway tunnel as an example. The tunnel crosssection is shown in Figure 2. The parameters for the calculation are $E_{1}=15 \mathrm{GPa} ; E_{2}=20 \mathrm{GPa} ; \mu_{1}=0.28 ; \mu_{2}=0.2 ; \eta=$ $0.6 ; p=6 \mathrm{MPa} ; \lambda=1$.

5.1. Accuracy of the Complex Function Method. The accuracy of the complex function method was highly related to the number of $\mathrm{Ne}$ and $\mathrm{Nf}$. The reality stress components $\sigma_{\rho}$ and $\tau_{\rho \theta}$ of the lining inner boundary equal zero. Thus, the accuracy of the proposed method can be examined by comparing the calculated $\sigma_{\rho}$ and $\tau_{\rho \theta}$ of the lining inner boundary with zero. The results of $\sigma_{\rho}$ and $\tau_{\rho \theta}$ at $L_{1}$ were shown in Figures $4-9$. As shown in Figures 4-9, the calculation precision was improved with the increase of the number of $\mathrm{Ne}$. When $\mathrm{Ne}$ equals to 10 or 20, the boundary condition at $L_{1}$ was not well satisfied with the reality. While $\mathrm{Ne}$ equals 30 , the absolute results of calculated normal stress $\sigma_{\rho}$ and shear stress $\tau_{\rho \theta}$ are far less than 0.001 . This means the complex variable method could be accurate enough to satisfy the boundary condition at $L_{1}$ when the $N e$ is greater than 30 .

The displacement components of lining and surrounding rock-mass were also presented in Figures 10 and 11. It can be concluded from the two figures that the displacement continuity condition was also well satisfied when $\mathrm{Ne}$ equals 


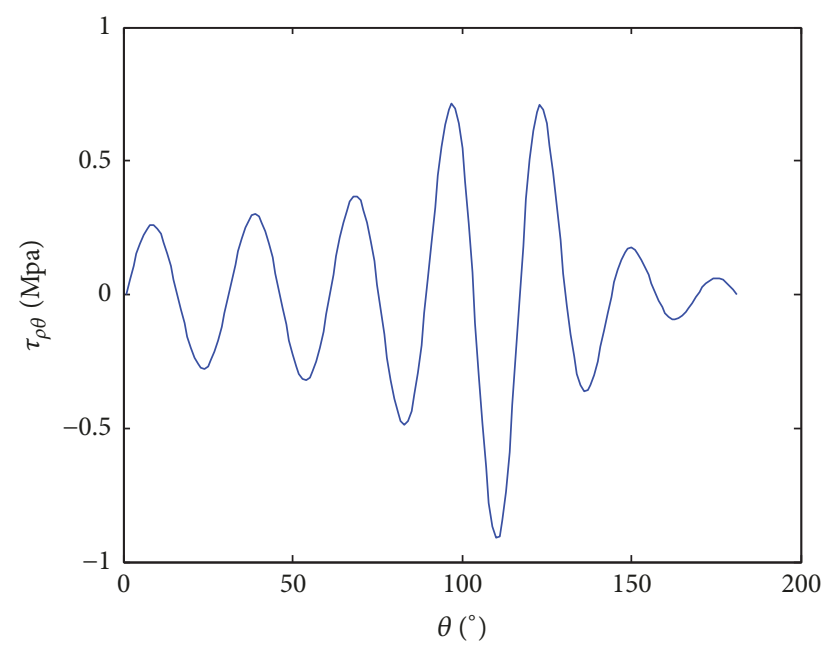

FIGURE 5: Shear stress of lining inner boundary $(\mathrm{Ne}=10)$.

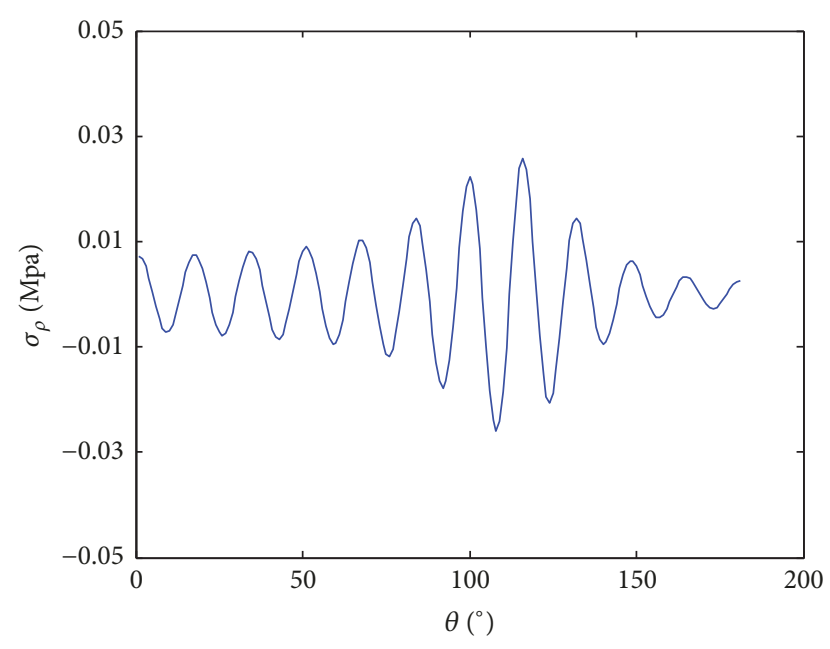

FIGURE 6: Normal stress of lining inner boundary $(\mathrm{Ne}=20)$.

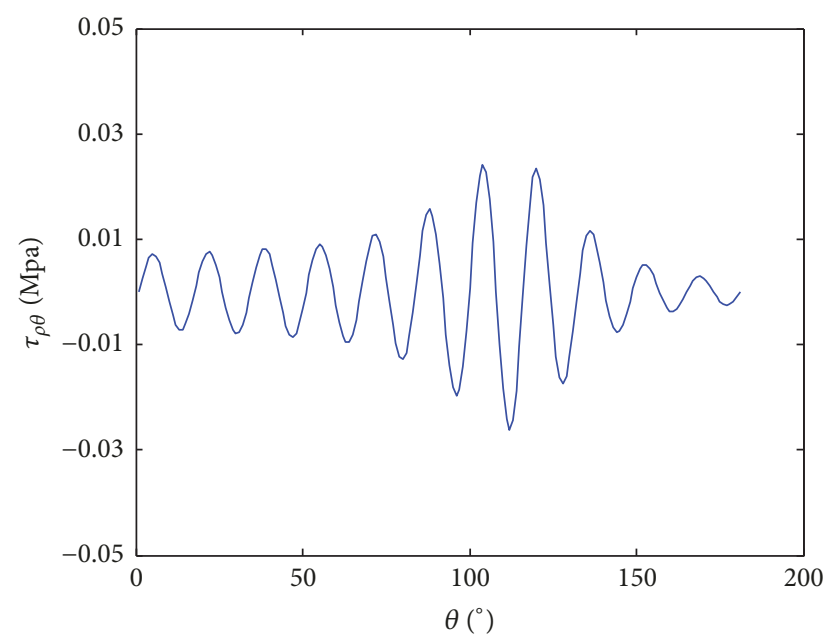

FIgURE 7: Shear stress of lining inner boundary $(\mathrm{Ne}=20)$.

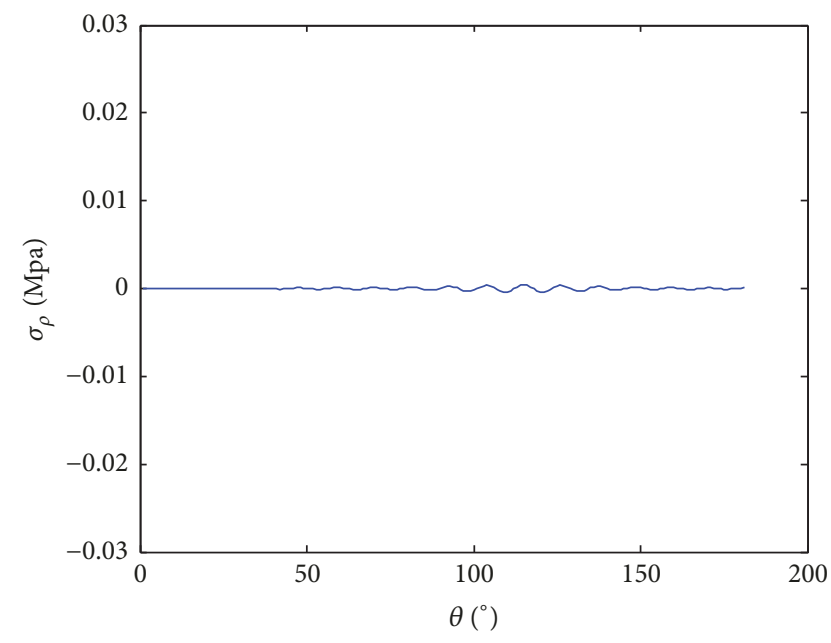

Figure 8: Normal stress of lining inner boundary $(\mathrm{Ne}=30)$.

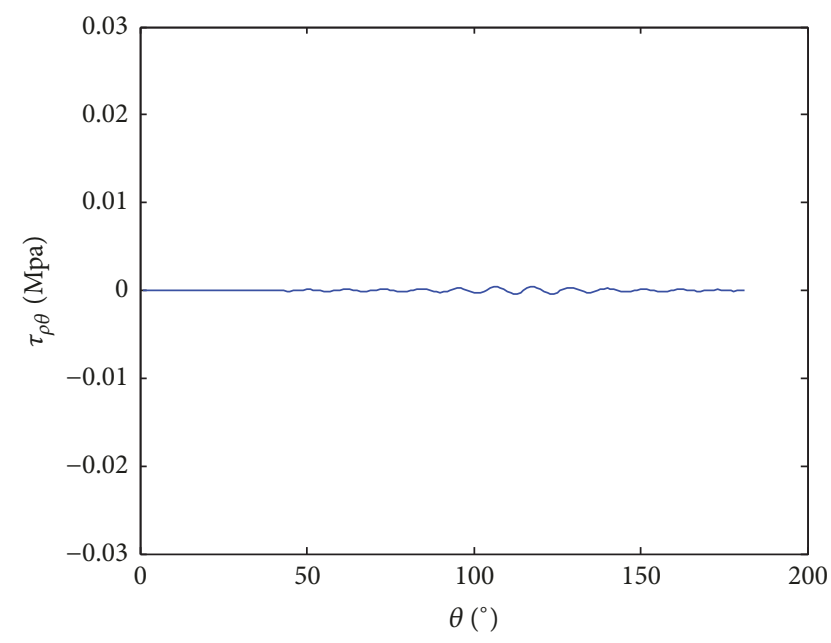

Figure 9: Shear stress of lining inner boundary $(\mathrm{Ne}=30)$.

30. Thus, 30 were chosen as the number of $N e$ in the following calculation.

5.2. Lining Forces and Discussion. The tangential stresses of the two boundaries of the lining were shown in Figure 12. Both tangential stresses showed the same trend. The left or right half of the tunnel lining possessed two peaks and two troughs in the tangential stress curve. The maximum value occurred at $115^{\circ}$ from the tunnel crown to the side wall. Another peak occurred at the tunnel crown. The troughs occurred at about $70^{\circ}$ from the tunnel crown to the side wall as well as the centre part of the tunnel invert. From Figure 13, both positive and negative bending moment existed in the lining. The three turning points of the positive and negative of the bending moment are $40^{\circ}, 95^{\circ}$, and $135^{\circ}$. Figure 14 is the $3 \mathrm{D}$ surface plot of the tangential stress in different depth of the lining. From the projection of the 3D surface on the $x-o-z$ plane $\left(\theta-o-\sigma_{\theta}\right.$ plane), the tangential stress of different layers of lining intersected each other at the same $\theta$ where the bending moment was zero. From the projection of the $3 \mathrm{D}$ 


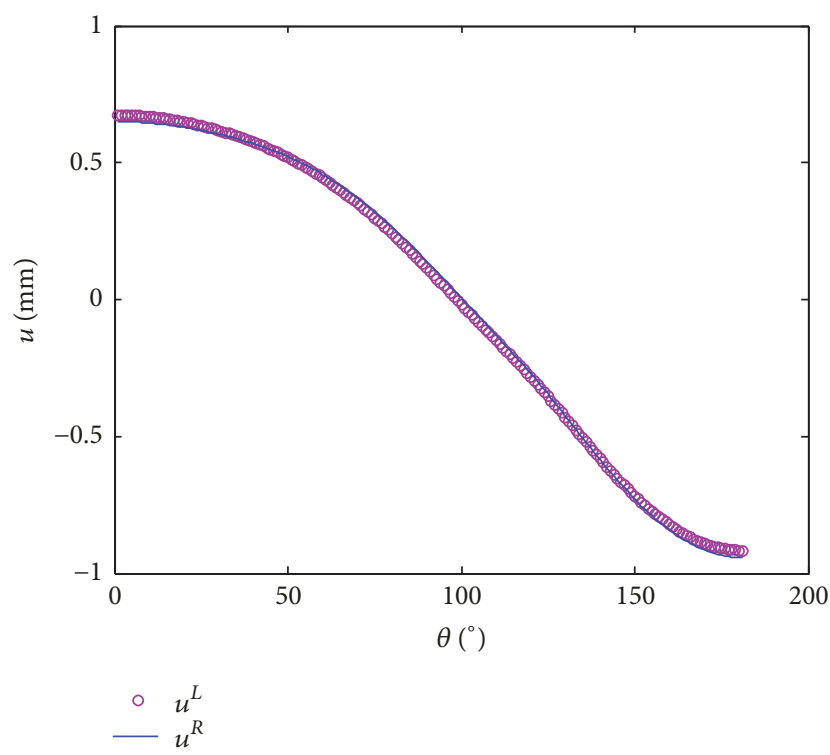

Figure 10: Displacement $u$ along $L_{2}(N e=30)$.

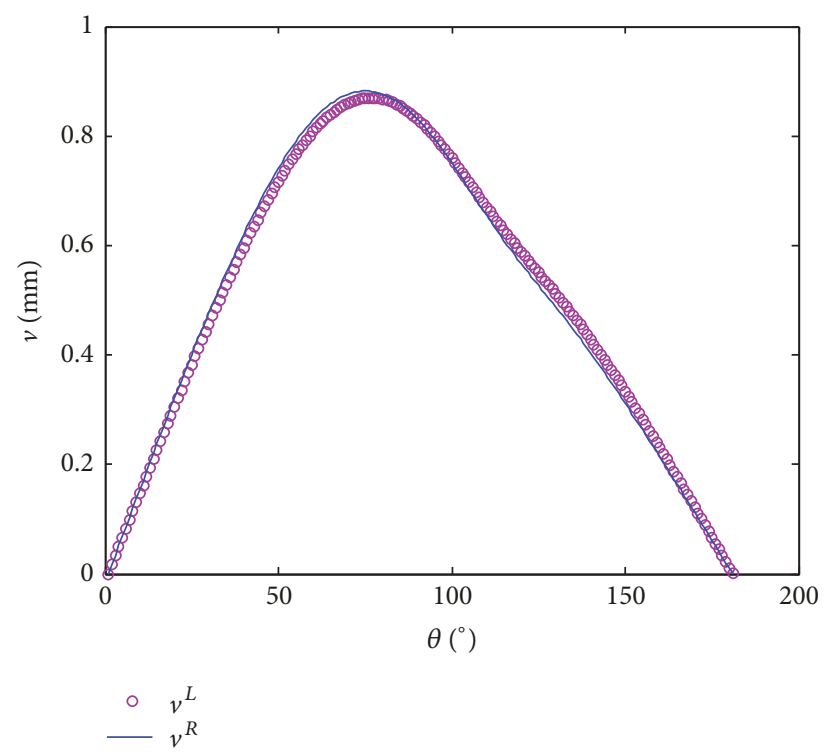

FIGURE 11: Displacement $v$ along $L_{2}(\mathrm{Ne}=30)$.

surface on the $y-o-z$ plane ( $t-o-\sigma_{\theta}$ plane), a linear relationship between lining depth and tangential stress could be found and provided evidence for the assumption in Section 4.2.

Figures 15 and 16 showed the relationship between the tangential stresses and the parameters $\theta$ and $\lambda$ at the two lining boundaries. It can be obtained that $\lambda$ had great influence on tangential stresses of the lining. From the projection on the $x-o-y$ plane, negative tangential stress occurred when $\lambda$ was smaller than 0.6. Thus, for tunnels to be built in rock with small lateral earth pressure coefficient (e.g., loess with a coefficient of lateral earth pressure equals 0.5 [21, 22]), reinforced concrete, and fibre concrete linings rather than plain concrete linings are recommended for the higher durability performance of the tunnel. From the projection

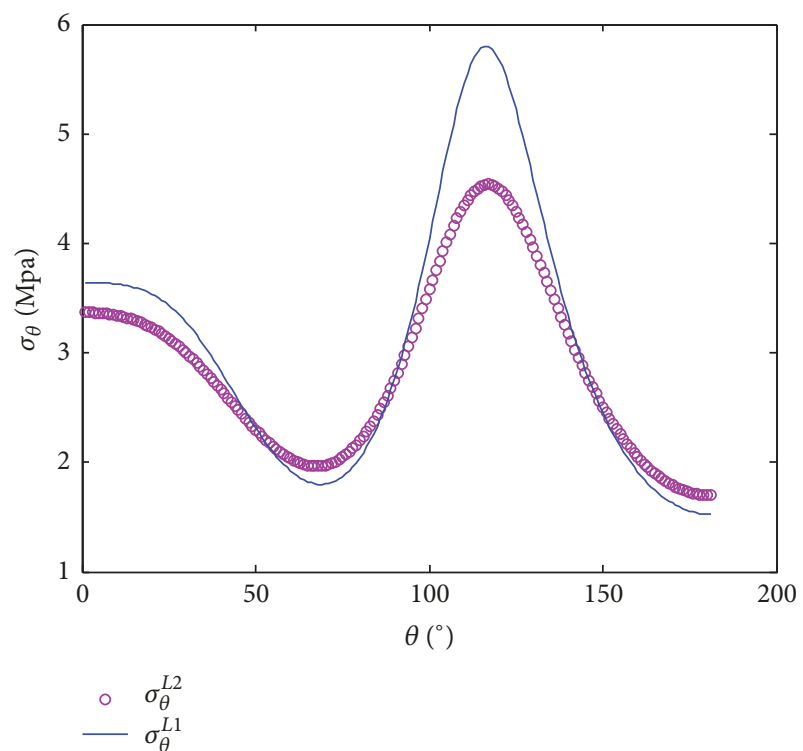

FIgURE 12: Tangential stress at the lining boundaries.

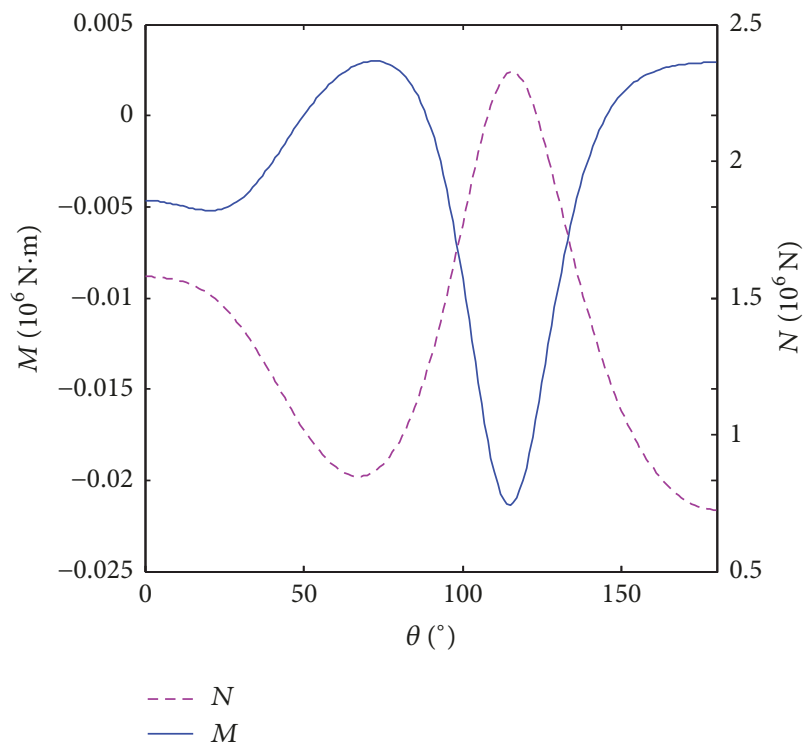

FIgURE 13: Axial force and bending moment.

on the $x-o-z$ plane, negative tangential stress occurred when $\theta$ was within $40^{\circ} \sim 90^{\circ}$. Therefore, tension-resistant design should be applied mainly to this part of lining.

The 3D surface projection on the $y-o-z$ plane showed a various linear relationship between the coefficient of lateral earth pressure and the tangential stress with different $\theta$.

5.3. Surrounding Rock-Mass Stress and Discussion. From Figure 17, the tangential stress declined sharply when $\rho$ was small than 2. In contrast, tangential stress was almost the same in regions where $\rho$ was great than 3 . Therefore, the tunnel excavation and lining support had a limited impact on the surrounding rock-mass. 


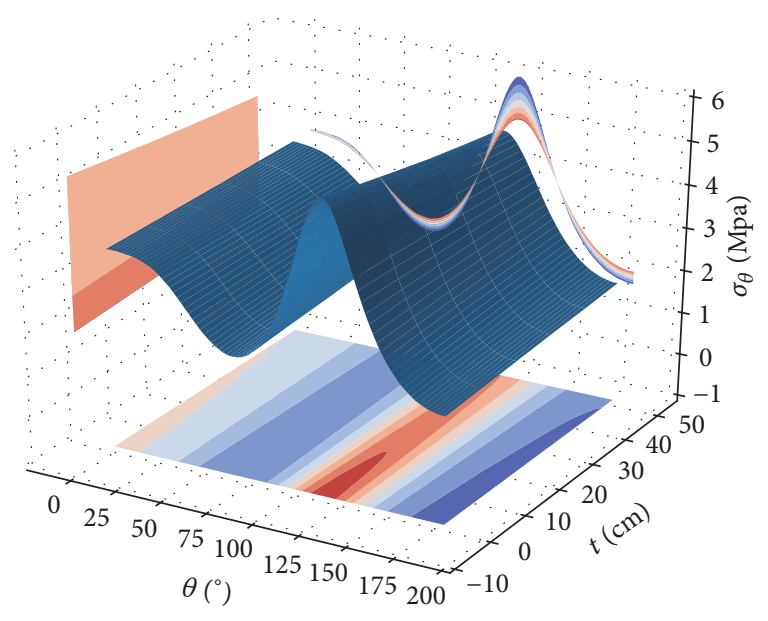

FIGURE 14: Tangential stress of the lining in different depth.

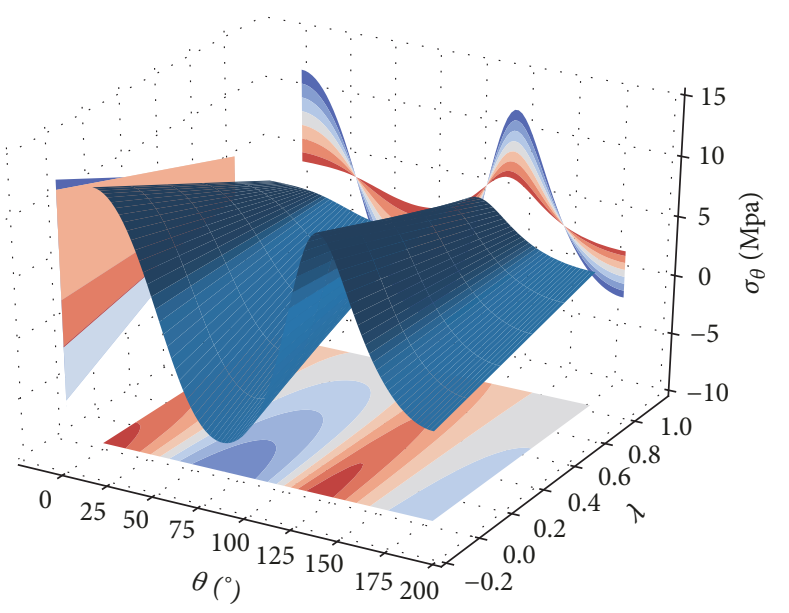

FIGURE 15: Tangential stress of inner boundary of the lining with $\theta$ and $\lambda$.

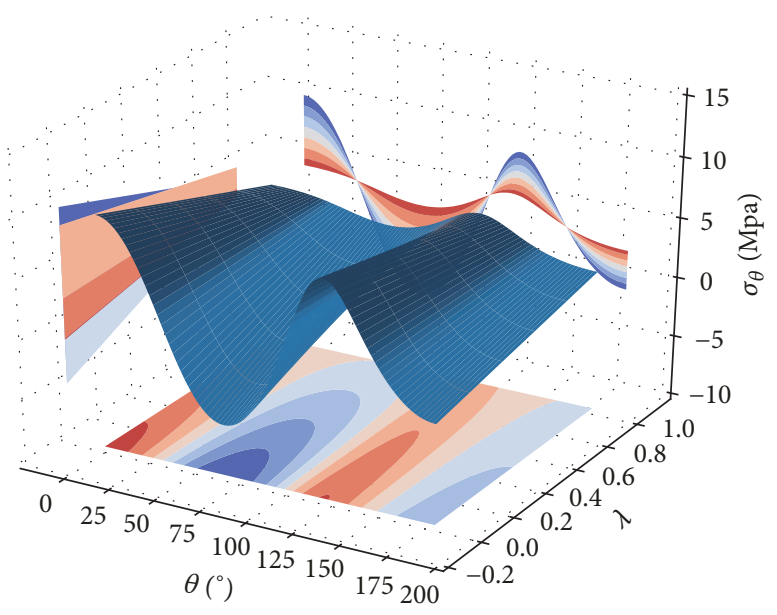

FIGURE 16: Tangential stress of outer boundary of the lining with $\theta$ and $\lambda$.

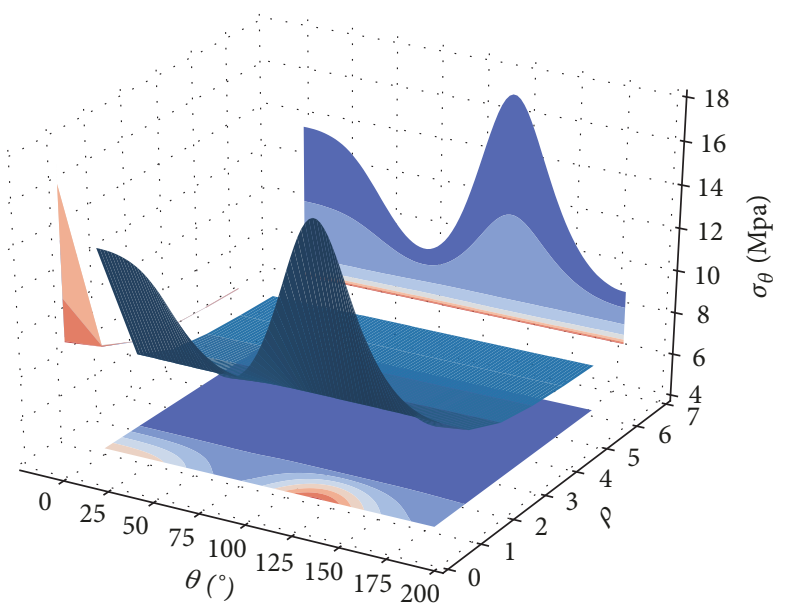

FIGURE 17: Relationship between tangential stress of rock-mass and parameters $\theta$ and $\rho$.

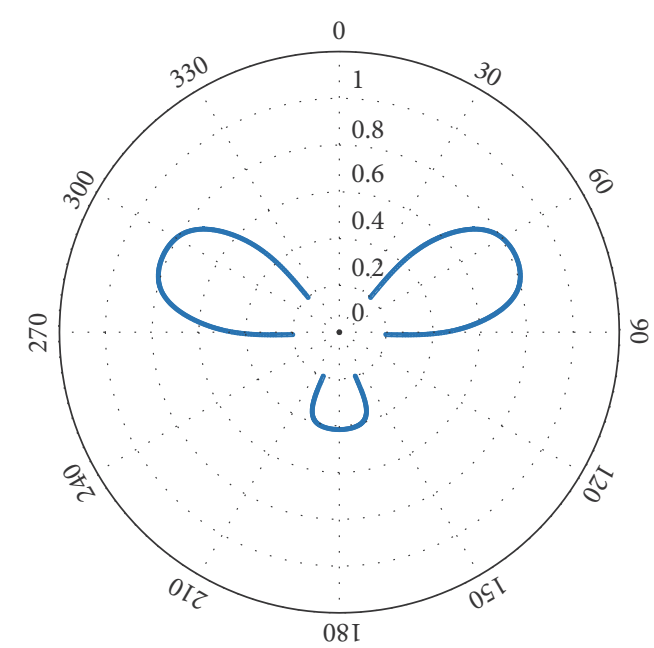

FIGURE 18: Relationship between $\theta$ and $\lambda$ of the interface from the rock side, while $\sigma_{\theta}=0$.

The surrounding rock-mass will be in an unstable state when the tangential stress equals zero [23]. The tangential stress of the interface from the rock side was calculated and shown in Figure 18. When the coefficient of the lateral pressure was between 0 and 0.6 , the zero value of the tangential stress occurred where $\theta$ is from $45^{\circ}$ to $90^{\circ}, 160^{\circ}$ to $200^{\circ}$, and $270^{\circ}$ to $315^{\circ}$. Therefore, supporting of the surrounding rock-mass in these regions should be enhanced to avoid the collapse during the tunnel excavation.

\section{Conclusion}

An elastic plane strain solution for MCA tunnels was presented based on the complex variable method. Stress and displacement components were predicted by employing complex potential functions which were consequently determined by Cauchy integration and series solution method. Force solutions for linings were also obtained according to the tangential stress at the two boundaries. 
The boundary conditions can be well satisfied with the reality when the number of terms of the analytic function is greater than 30 . As to the linings, the maximum value of the tangential stress occurs at the corner of the side wall. The negative tangential stress occurs in tunnels with small lateral earth pressure coefficients, especially at regions from the tunnel shoulder to the side wall. A linear relationship was shown between the tangential stress and the lining depth. Also, the change of tangential stress is proportional to the coefficient of lateral earth pressure, but the slope varies with angles from the tunnel crown to the tunnel side wall. As to the surrounding rock-mass, the tunnel excavation and lining support have limited influence on the region when $\rho$ is less than 2 . When the coefficient of the lateral pressure is between 0 and 0.6 , the zero value of the tangential stress occurs in the section from the tunnel shoulder to the side wall as well as the middle part of the invert. Thus, during the tunnel excavation, the addition of surrounding rock-mass in these regions should be considered to avoid the collapse accidents.

The difference existing before and after the conformal mapping of the lining outline also has an influence on correctness of results. More attempts should be made in upcoming works to improve the accuracy of conformal mapping functions and preserve shape after mapping.

\section{Data Availability}

The data used to support the findings of this study are included within the article.

\section{Conflicts of Interest}

The authors declare that they have no conflicts of interest.

\section{Acknowledgments}

The research work reported herein was made possible by the China Scholarship Council (no. 201706560008). The financial support is highly appreciated. The authors would also like to express special thanks to Professor Ai-Zhong Lu from North China Electric Power University and Professor Wei Gao and Dr. Kang Gao from University of New South Wales for their assistance during the derivation works.

\section{References}

[1] Y. Tan, J. V. Smith, C.-Q. Li, M. Currell, and Y. Wu, "Predicting external water pressure and cracking of a tunnel lining by measuring water inflow rate," Tunnelling and Underground Space Technology, vol. 71, pp. 115-125, 2018.

[2] Y. M. A. Hashash, J. J. Hook, B. Schmidt, and J. I-Chiang Yao, "Seismic design and analysis of underground structures," Tunnelling and Underground Space Technology, vol. 16, no. 4, pp. 247-293, 2001.

[3] C. Zhao, A. Alimardani Lavasan, T. Barciaga, C. Kämper, P. Mark, and T. Schanz, "Prediction of tunnel lining forces and deformations using analytical and numerical solutions," Tunnelling and Underground Space Technology, vol. 64, pp. 164176, 2017.
[4] S. C. Möller and P. A. Vermeer, "On numerical simulation of tunnel installation," Tunnelling and Underground Space Technology, vol. 23, no. 4, pp. 461-475, 2008.

[5] X. Liu, Y. Liu, Z. Yang, and C. He, "Numerical analysis on the mechanical performance of supporting structures and ground settlement characteristics in construction process of subway station built by Pile-Beam-Arch method," KSCE Journal of Civil Engineering, vol. 21, no. 5, pp. 1690-1705, 2017.

[6] V. Avgerinos, D. M. Potts, and J. R. Standing, "Numerical investigation of the effects of tunnelling on existing tunnels," Geotechnique, vol. 67, no. 9, pp. 808-822, 2017.

[7] G. Behnen, T. Nevrly, and O. Fischer, "Soil-structure interaction in tunnel lining analyses," Geotechnik, vol. 38, no. 2, pp. 96-106, 2015.

[8] Y.-L. Pi, M. A. Bradford, and B. Uy, "In-plane stability of arches," International Journal of Solids and Structures, vol. 39, no. 1, pp. 105-125, 2001.

[9] W. Gao, D. Wu, K. Luo, and Y.-L. Pi, "Stochastic Behaviour of Shallow Concrete-filled Steel Tubular Arches," in Proceedings of the 23rd Australasian Conference on the Mechanics of Structures and Materials (ACMSM23), pp. 663-668, Byron Bay, Australia, 2014.

[10] S. G. Lekhnitskii, Anisotropic plates, Foreign Technology Div Wright-Patterson Afb Oh, 1968.

[11] A. M. Hefny and K. Y. Lo, "Analytical solutions for stresses and displacements around tunnels driven in cross-anisotropic rocks," International Journal for Numerical and Analytical Methods in Geomechanics, vol. 23, no. 2, pp. 161-177, 1999.

[12] T. M. Vu, J. Sulem, D. Subrin, and N. Monin, "Semi-analytical solution for stresses and displacements in a tunnel excavated in transversely isotropic formation with non-linear behavior," Rock Mechanics and Rock Engineering, vol. 46, no. 2, pp. 213229, 2013

[13] X. Han, L. Chai, and Y. Xia, "Theoritical analysis of the deformation of shield tunnel segment under fire situation," Chinese Journal of Underground Space and Engineering, vol. 13, no. 2, pp. 525-530, 2017.

[14] J. Lai, S. Mao, J. Qiu et al., "Investigation progresses and applications of fractional derivative model in geotechnical engineering," Mathematical Problems in Engineering, vol. 2016, Article ID 9183296, 15 pages, 2016.

[15] F. Ye, C. F. Gou, H. D. Sun, Y. P. Liu, Y. X. Xia, and Z. Zhou, "Model test study on effective ratio of segment transverse bending rigidity of shield tunnel," Tunnelling and Underground Space Technology, vol. 41, no. 1, pp. 193-205, 2014.

[16] Y. H. Takano and I. T. A. Working Grp, "Guidelines for the design of shield tunnel lining," Tunnelling And Underground Space Technology, vol. 15, no. 3, pp. 303-331, 2000.

[17] A.-Z. Lu, L.-Q. Zhang, and N. Zhang, "Analytic stress solutions for a circular pressure tunnel at pressure and great depth including support delay," International Journal of Rock Mechanics and Mining Sciences, vol. 48, no. 3, pp. 514-519, 2011.

[18] N. Yasuda, K. Tsukada, and T. Asakura, "Elastic solutions for circular tunnel with void behind lining," Tunnelling and Underground Space Technology, vol. 70, pp. 274-285, 2017.

[19] N. I. Muskhelishvili and J. R. M. Radok, Some Basic Problems of the Mathematical Theory of Elasticity, Cambridge Univ Press, London, UK, 1953.

[20] A.-Z. Lu, N. Zhang, and L. Kuang, "Analytic solutions of stress and displacement for a non-circular tunnel at great depth including support delay," International Journal of Rock Mechanics and Mining Sciences, vol. 70, pp. 69-81, 2014. 
[21] J. X. Niu and D. L. Xie, "Coefficient of at rest earth pressure of collapsible loess," Soil Engineering and Foundation, vol. 29, no. 4, pp. 124-126, 2015.

[22] J. Qiu, Y. Xie, H. Fan, Z. Wang, and Y. Zhang, "Centrifuge modelling of twin-tunnelling induced ground movements in loess strata," Arabian Journal of Geosciences, vol. 10, no. 22, 2017.

[23] F. Cui, L. Wang, and Y.-F. Wang, "Stress Analytic solution for elastic surrounding rock mass of circular tunnels subjected to non axisymmetric loads," Chinese Journal of Underground Space and Engineering, vol. 13, no. 3, pp. 637-642, 2017. 


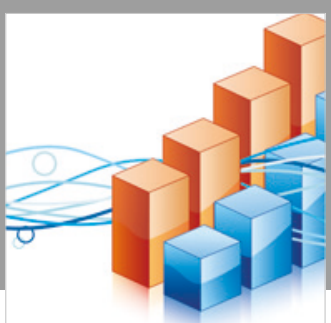

Advances in

Operations Research

\section{-n-m}
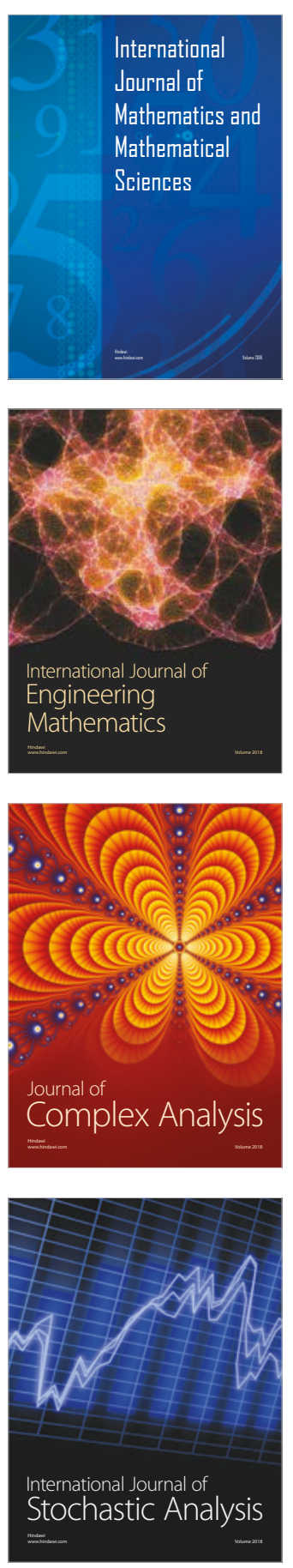
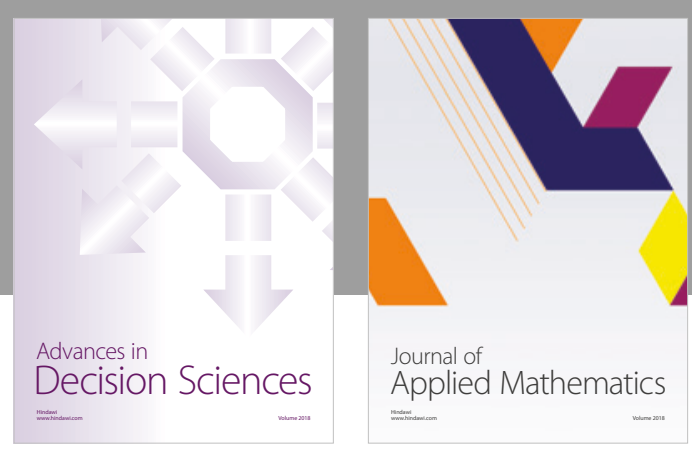

Journal of

Applied Mathematics
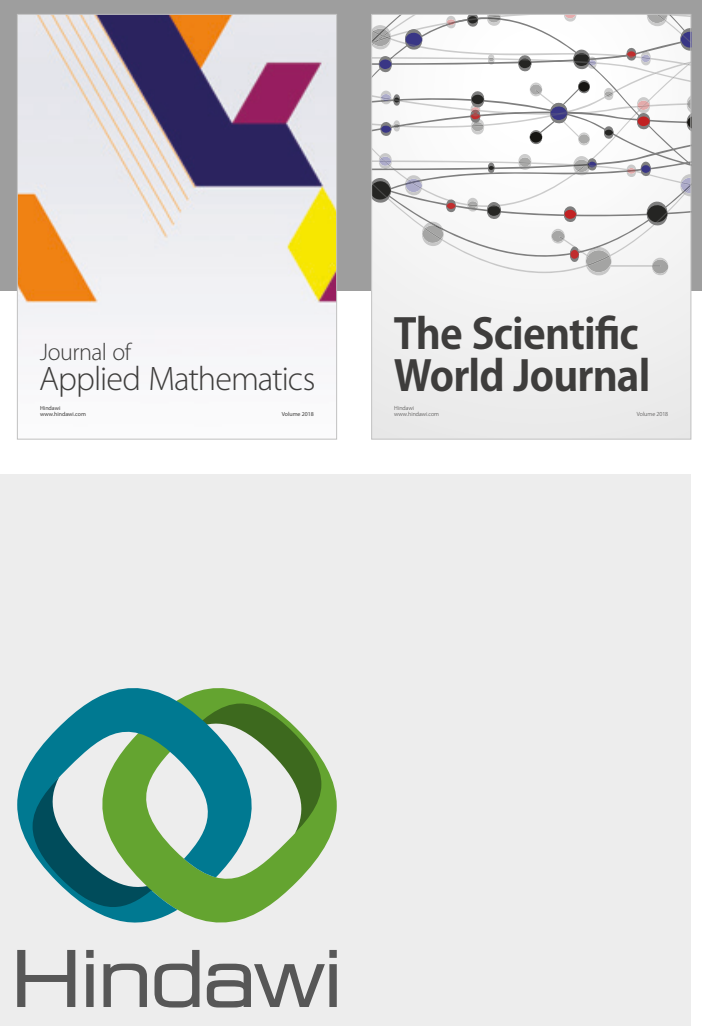

Submit your manuscripts at

www.hindawi.com

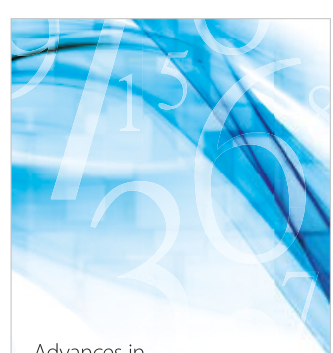

Advances in
Numerical Analysis
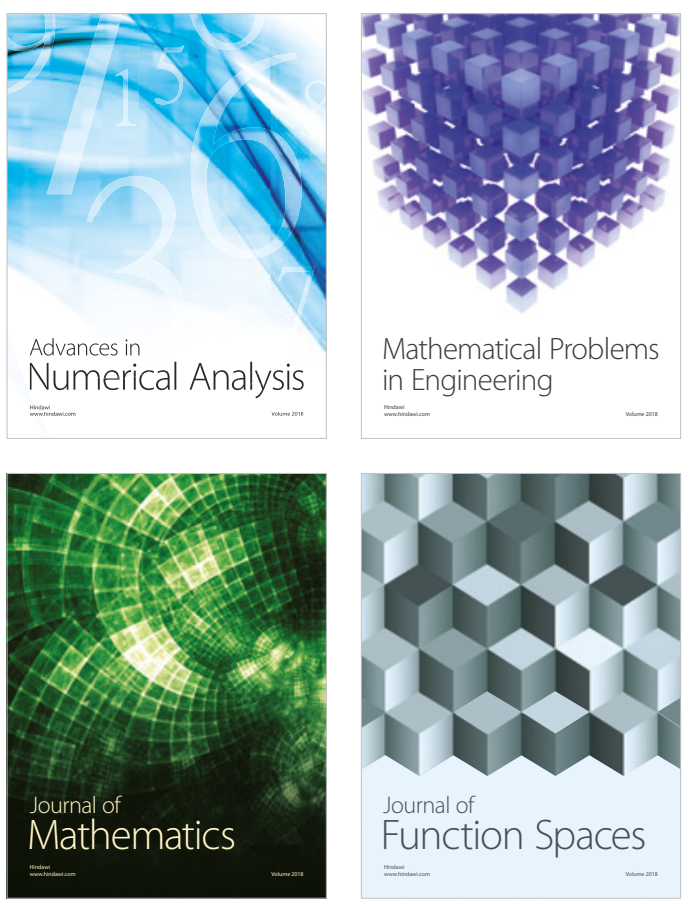

Mathematical Problems in Engineering

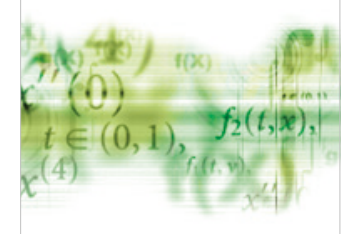

International Journal of

Differential Equations

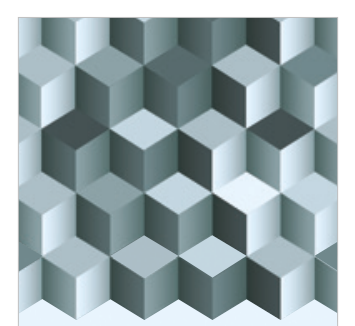

Journal of

Function Spaces

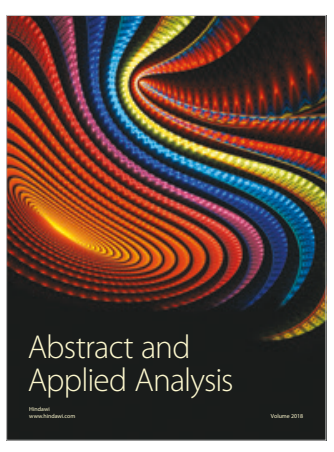

The Scientific

World Journal

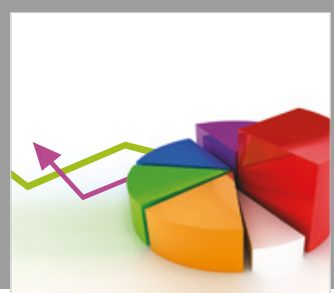

Journal of

Probability and Statistics
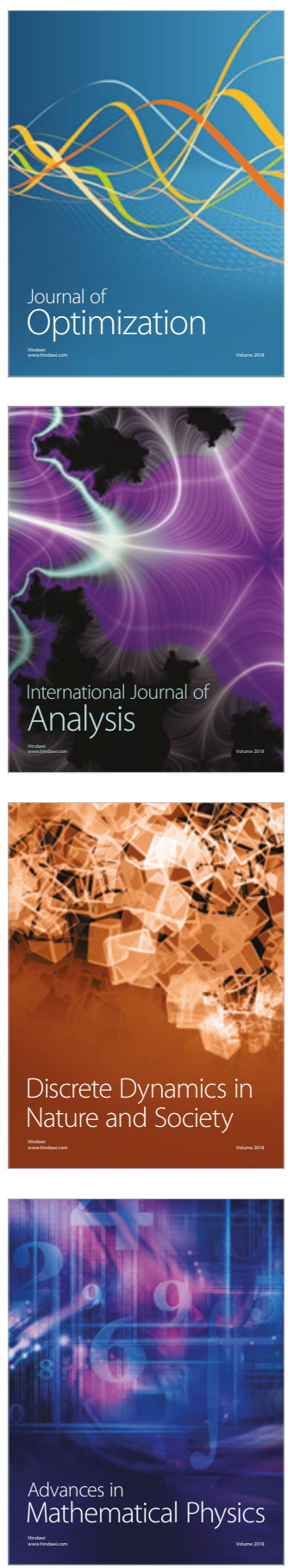